\title{
Efficiency and equilibria in games of optimal derivative design
}

\author{
Horst, Ulrich ; Moreno-Bromberg, Santiago
}

\begin{abstract}
In this paper, optimal derivative design when multiple firms compete for heterogenous customers is studied. Ties in the agents' best responses generate discontinuous payoffs. Efficient tie-breaking rules are considered: In a first step, the model presented by Carlier etal. (Math Financ Econ 1:57-80, 2007) is extended, and results of Page and Monteiro (J Math Econ 39:63-109, 2003, J Econ Theory 134:566-575, 2007, Econ Theory 34:503-524, 2008) are used to prove the existence of (mixed-strategies) Nash equilibria. In a second step, the case of risk minimizing firms is studied. Socially efficient allocations are introduced, and their existence is proved. In particular, the entropic risk measure is considered
\end{abstract}

DOI: https://doi.org/10.1007/s11579-012-0066-5

Posted at the Zurich Open Repository and Archive, University of Zurich ZORA URL: https://doi.org/10.5167/uzh-156738

Journal Article

Published Version

Originally published at:

Horst, Ulrich; Moreno-Bromberg, Santiago (2011). Efficiency and equilibria in games of optimal derivative design. Mathematics and Financial Economics, 5(4):269-297.

DOI: https://doi.org/10.1007/s11579-012-0066-5 


\title{
Efficiency and equilibria in games of optimal derivative design
}

\author{
Ulrich Horst · Santiago Moreno-Bromberg
}

Received: 5 July 2011 / Accepted: 29 February 2012 / Published online: 30 March 2012

(C) Springer-Verlag 2012

\begin{abstract}
In this paper, optimal derivative design when multiple firms compete for heterogenous customers is studied. Ties in the agents' best responses generate discontinuous payoffs. Efficient tie-breaking rules are considered: In a first step, the model presented by Carlier et al. (Math Financ Econ 1:57-80, 2007) is extended, and results of Page and Monteiro (J Math Econ 39:63-109, 2003, J Econ Theory 134:566-575, 2007, Econ Theory 34:503-524, 2008) are used to prove the existence of (mixed-strategies) Nash equilibria. In a second step, the case of risk minimizing firms is studied. Socially efficient allocations are introduced, and their existence is proved. In particular, the entropic risk measure is considered.
\end{abstract}

Keywords Adverse selection · Competing mechanisms · Delegation principle · Risk sharing $\cdot$ Pareto optimality

JEL Classification $\quad \mathrm{C} 62 \cdot \mathrm{C} 72 \cdot \mathrm{D} 43 \cdot \mathrm{D} 82 \cdot \mathrm{G} 14$

\section{Introduction}

In the standard Principal-Agent model, a monopolist (the principal) contracts with heterogeneous customers (the agents) on take-it-or-leave-it basis. Heterogeneity is usually captured by types. An agent's type is private information; the principal is aware of the distribution of types, but he cannot distinguish different agents prior to engaging them. This prevents the principal from extracting the maximum indifference price from each agent type. Instead, he designs incentive compatible catalogues with the intention of, at least partially, screening the

U. Horst

Institut für Mathematik, Humboldt-Universität zu Berlin, Unter den Linden 6, 10099 Berlin, Germany

e-mail: horst@math.hu-berlin.de

S. Moreno-Bromberg $(\varangle)$

Institut für Banking und Finance, Universität Zürich, Plattenstraße 32, 8032 Zurich, Switzerland

e-mail: santiago.moreno@bf.uzh.ch 
market. The characterization of the solutions to problems of this kind can be found, among others, in the works of Armstrong [2], Mussa and Rosen [15] and Rochet and Choné [23].

This paper considers a model of oligopolistic competition among providers of contingent claims under adverse selection. Two main challenges emerge when different firms compete for the business of heterogenous agents: the Revelation Principle no longer fully applies and ties in the agents' best-response mappings may emerge. When agents are indifferent between contracting with different firms, these firms' payoff functions may have discontinuities. This precludes the use of classical results (see for example [10]) to guarantee the existence of Nash equilibria in such games. Our main focus will therefore be on efficient allocations of risk, i.e. on allocations that maximize the combined performance of the firms.

We first extend the model of profit-maximizing firms presented in [6] to a multi-firm one. Here the theory of games played under adverse selection comes into play. In order to use recent results from the theory of catalogue games, we assume that each firm's strategy set consists of the closed, convex hull of a finite number of basic products. We show that when the game is played under a particular kind of tie-breaking rules, which paraphrasing [20] we call efficient, then it is uniformly payoff secure and reciprocally upper semicontinuous and hence has a mixed-strategies Nash equilibrium.

Efficient tie-breaking rules also play a key role in our multi-firm extension of the risk minimization model studied in [11]. In both cases the impact of a single trade on the firm's risk evaluation is highly non-linear and depends strongly on the firm's overall position. ${ }^{1}$ This results in two considerable technical difficulties. First, there is no reason to expect that the firms' payoff functions will be uniformly payoff secure. ${ }^{2}$ Second, most of the current results (e.g. $[3,10,18,16])$ on existence of Nash equilibria require some form of quasiconcavity. The exception is [24], where a complete, yet very challenging to verify, characterization of Nash equilibria is presented. In our model these conditions are only satisfied by the mixed extension of the game. Even if the game were uniformly payoff secure and weakly reciprocally upper semicontinuous, which is by no means a given, ${ }^{3}$ considering a mixed extension of the game would mean the following: firms would view linear aggregation of possible risk evaluations as the way to assess the influence of others' in their own risk. Whether this approach is consistent with the ideas behind the theory of convex risk measure is in our opinion debatable. Taking the previous arguments into account, we do not seek the existence of mixed-strategies Nash equilibria. Instead we establish existence socially efficient allocations of risk. Such allocations minimize the firms' aggregate risk. As such, our work can be viewed as a first attempt to extend the seminal paper of Jouini et al. [12] on efficient risk sharing to an asymmetric information framework.

In terms of implementability, we show for the case of the entropic risk measure that among all efficient tie-breaking-rules, one is a constant ratio of market shares. In other words, there is a "fix-mix" ratio under which firms share the whole market, rather than segmenting it by agent types. A real-world example where firms offer consumers essentially the same utility (using different products), and where the assumption of mean-variance optimizing consumers is appropriate, is retail banking. We illustrate this result with a numerical example, in which we also find that (as expected) competition benefits buyers. The numerical algorithm

\footnotetext{
1 In a model of profit maximization a firm contracts with some agent type independently of other types as long as that particular type contributes positively to the firm's revenues.

2 The "worst-case" tie-breaking rule, where each firm assumes ties will be broken in the most disadvantageous way for itself, does yield payoff security, but at the cost of reciprocally upper semicontinuity.

3 This would imply the existence of Nash equilibria in mixed strategies
} 
that we use for this example, a hybrid descent method, is also used to analyze an example where the firms are AV@R-minimizers.

The remainder of this paper is organized as follows: Sect. 2 contains the description of our general framework: The asymmetry of information in the model, the best-response sets of the agents that give rise to ties, and the role of tie-breaking rules are studied. In Sect. 3 we analyze the game played among profit-maximizing firms, where the main result is the existence of mixed-strategies Nash equilibria. Section 4 is devoted to the risk-minimization game, where we prove the existence of socially efficient allocations. Finally, we present in Sect. 5 numerical algorithms to estimate equilibrium points and socially efficient allocations in some particular examples, as well as the "fix-mix" result mentioned above. The proofs of auxiliary lemmas and propositions can be found in the Appendix.

\section{General framework}

We consider two firms and a continuum of agents. The firms sell structured products to the agents ${ }^{4}$. We study both profit maximizing and risk minimizing firms. In the profit-maximization case, the firms maximize expected income from trading with the agents; the impact of on individual trade on the firm's objective function is hence linear. In the risk-minimization case, the firms evaluate their overall position using convex risk measures; in this case the impact of an individual trade on a firm's objective function is non-linear. The precise mathematical formulations of the problems at hand require the concepts contained in this section.

\subsection{The financially feasible sets}

Firms compete by offering the agents derivatives contracts. The financially feasible set for firm $i=1,2$ is

$$
\mathcal{X}_{i} \subset \mathbb{L}^{2}(\Omega, \mathcal{F}, \mathbb{P}),
$$

where $(\Omega, \mathcal{F}, \mathbb{P})$ is a standard probability space. We assume these sets are closed, convex and bounded, and that $0 \in \mathcal{X}_{i}$. Any additional requirements on the sets $\mathcal{X}_{i}$ shall be introduced when necessary. ${ }^{5}$ In the literature on multi-firm, non-linear pricing games, it is generally assumed that each firm chooses a compact subset $\mathcal{Y}_{i}$ from its financially feasible set, and it devises a (non-linear) pricing schedule

$$
p_{i}: \mathcal{Y}_{i} \rightarrow \mathbb{R}
$$

Each pair $\left(X, p_{i}(X)\right)$ (where $\left.X \in \mathcal{X}_{i}\right)$ is called a contract. The principles of delegation and competitive taxation (see for example [18]) allow us, with no loss of generality, to restrict our study of existence of equilibria to games played over compact subsets of the product-price space $\mathcal{X}_{i} \times \mathbb{R}$ (these are known as catalogue games). The boundedness assumption on $\mathcal{X}_{i}$ implies that prices belong to some compact set $P \subset \mathbb{R}$. We write $C_{i}$ to denote a catalogue offered by firm $i$, and $\left(C_{1}, C_{2}\right)$ for a catalogue profile (also called a market situation).

\footnotetext{
4 We have over-the-counter trading in mind.

5 We omit writing $i=1,2$ when we refer to properties shared by both firms.
} 


\subsection{The agents' preferences}

The agents are heterogenous, mean-variance maximizers whose set of types is

$$
\Theta=[\underline{\theta}, \bar{\theta}] \text { for some } \underline{\theta}>0 .
$$

An agent's type represents his risk aversion: given a contingent claim $Y$ an agent of type $\theta$ assesses its worthiness via the (type-dependent) utility function

$$
U(\theta, Y)=\mathrm{E}[Y]-\theta \operatorname{Var}[Y] .
$$

The types are private information. They are distributed according to a measure $\mu$, which is common knowledge, and it is absolutely continuous with respect to Lebesgue measure. This asymmetry of information, also known as adverse selection, prevents the firms from extracting all the above-reservation-utility wealth form each agent. The knowledge of $\mu$ is therefore essential for the firms. ${ }^{6}$ In the upcoming sections we require the following auxiliary lemma, whose proof is a straightforward application of the Cauchy-Schwartz inequality:

Lemma 2.1 The family of functions $\mathcal{U}=\{U(\theta, \cdot) \mid \theta \in \Theta\}$ is uniformly equicontinuous.

\subsection{Indirect utilities and best-response sets}

When an agent faces a catalogue profile $\left(C_{1}, C_{2}\right)$, he chooses a single contract from some $C_{i}$ on a take-it-or-leave-it basis. Given $\left(C_{1}, C_{2}\right)$, the indirect utility of an agent of type $\theta$ is: ${ }^{7}$

$$
v\left(\theta, C_{1}, C_{2}\right) \triangleq \sup \left\{U(\theta, X)-p \mid(X, p) \in C_{i} \text { for some } i\right\} .
$$

We assume that the agents have an outside option that yields their reservation utility, which we normalize to zero. For a fixed market situation, the function $v\left(\cdot, C_{1}, C_{2}\right): \Theta \rightarrow \mathbb{R}$ is convex, being the pointwise supremum of affine functions of $\theta$. The presence the outside option guarantees $v \geq 0$. The best-response set of the agents of type $\theta$ to a certain catalogue profile is defined as:

$$
\mathcal{L}\left(\theta, C_{1}, C_{2}\right) \triangleq \operatorname{argsup}\left\{U(\theta, X)-p \mid(X, p) \in C_{i} \text { for some } i\right\} .
$$

In Sects. 3 and 4 we make necessary assumptions on $C_{i}$ as to guarantee $\mathcal{L}\left(\theta, C_{1}, C_{2}\right) \neq \emptyset$ for all $\theta \in \Theta$. The Envelope Theorem (see for example [14]) implies that for any $(X(\theta), p(\theta)) \in$ $\mathcal{L}\left(\theta, C_{1}, C_{2}\right)^{8}$

$$
v^{\prime}\left(\theta, C_{1}, C_{2}\right)=-\operatorname{Var}[X(\theta)] \quad \mu-\text { a.s.. }
$$

In particular $v^{\prime}\left(\cdot, C_{1}, C_{2}\right) \leq 0 \mu$-a.s.. Equation (1) provides a valuable link between optimal contracts (from the point of view of the agents) and the indirect utility functions. In order to exploit the information contained in $\mu$, firms must choose catalogues that do not offer the agents incentives to lie about their types. The subset $C_{i}(\theta) \subset C_{i}$ of products that firm $i$ expects that agents of type $\theta$ will choose must satisfy

$$
\begin{aligned}
& \mathrm{E}[X(\theta)]-\theta \operatorname{Var}[X(\theta)]-p(\theta) \geq \mathrm{E}[Y]-\theta \operatorname{Var}[Y]-q, \quad \forall(X(\theta), p(\theta)) \in C_{i}(\theta), \\
& (Y, q) \in C_{i} .
\end{aligned}
$$

\footnotetext{
6 We could make additional assumptions on $\mu$ and allow for $\underline{\theta}=0$.

7 Our arguments can be easily extended to utility functions of the form $U(\theta, X, p)=\theta \phi_{1}(X)+\phi_{2}(X)-t$. What we require is that $U$ is affine in types and linear in transfers.

8 This envelope structure, which would read $v^{\prime}\left(\theta, C_{1}, C_{2}\right)=\phi_{1}(X(\theta))$ in the setting mentioned in Eq. 1, is central to our analysis.
} 
Catalogues that satisfy this property are called incentive compatible. Let

$$
\mathcal{L}\left(\theta, C_{i}\right) \triangleq \operatorname{argsup}\left\{U(\theta, X)-p \mid(X, p) \in C_{i}\right\},
$$

then $C_{i}$ is incentive compatible if and only if $C_{i}(\theta)=\mathcal{L}\left(\theta, C_{i}\right) .{ }^{9}$ A catalogue where the products intended for agents of type $\theta$ yield at least their reservation utility is said to be individually rational. Since all reservation utilities are zero, an individually rational catalogue satisfies

$$
\mathrm{E}[X(\theta)]-\theta \operatorname{Var}[X(\theta)]-p(\theta) \geq 0 \quad \forall(X(\theta), p(\theta)) \in \mathcal{L}\left(\theta, C_{i}\right) .
$$

\subsection{Tie-breaking rules and market segmentation}

A crucial feature of multi-firm games is the presence of ties. Since no convexity assumption is made on the catalogues' structure, the sets $\mathcal{L}\left(\theta, C_{1}, C_{2}\right)$ and $\mathcal{L}\left(\theta, C_{i}\right)$ need not be singletons. There can be both inter- and intra-firms ties, i.e. an agent may be indifferent between two products that are offered by distinct firms, or maybe between two contracts that are offered by the same firm. In what follows we study mechanisms via which ties are broken, as well as the partitions of $\Theta$ that are generated by tie-breaking. Given a catalogue profile $\left(C_{1}, C_{2}\right)$, we define

$$
v\left(\theta, C_{i}\right) \triangleq v_{i}(\theta) \triangleq \sup \left\{U(\theta, X)-p \mid(X, p) \in C_{i}\right\} .
$$

The $v_{i}$ 's are related to $v\left(\theta, C_{1}, C_{2}\right)$ via $v\left(\theta, C_{1}, C_{2}\right)=\max \left\{v\left(\theta, C_{1}\right), v\left(\theta, C_{2}\right)\right\}$. Some important properties of the functions $v\left(\theta, C_{i}\right)$ are summarized in Lemma 2.2 below, which follows from Proposition 2.2 in [6].

Lemma 2.2 Let $\left(C_{1}, C_{2}\right)$ be a catalogue profile and assume $\mathcal{L}\left(\theta, C_{i}\right) \neq \emptyset$ for all $\theta \in \Theta$, then:

1. The functions $v\left(\cdot, C_{i}\right): \Theta \rightarrow \mathbb{R}$ are convex.

2. If $C_{i}$ is incentive compatible then $-\operatorname{Var}\left[X_{i}(\theta)\right] \in \partial v\left(\theta, C_{i}\right)$ for all $\left(X_{i}(\theta), p_{i}(\theta)\right) \in$ $C_{i}(\theta)$. In particular, the equation $-\operatorname{Var}\left[X_{i}(\theta)\right]=v^{\prime}\left(\theta, C_{i}\right)$ holds $\mu$-a.s..

If for $\theta_{0} \in \Theta$ we have $v_{i}\left(\theta_{0}\right)>v_{-i}\left(\theta_{0}\right)$, then the agents of type $\theta_{0}$ will contract with firm $i$. The set of types that are indifferent between the firms' offers is

$$
\Theta_{0} \triangleq\left\{\theta \in \Theta \mid v\left(\theta, C_{1}\right)=v\left(\theta, C_{2}\right)\right\} .
$$

To avoid ambiguities we assume that if $v_{i}(\theta)=v_{-i}(\theta)=0$ then the corresponding agents opt for the outside option. The market is segmented into the sets

$$
\Theta_{1} \triangleq\left\{v_{1}>v_{2}\right\}, \quad \Theta_{2} \triangleq\left\{v_{2}>v_{1}\right\} \text { and } \Theta_{0} .
$$

In order to deal with types in $\Theta_{0}$ whose indirect utility is not zero, we define the set of tie-breaking rules (henceforth TBRs) as

$$
F \triangleq\left\{f \in \mathbb{L}^{0}[a, 1] \mid 0 \leq f \leq 1\right\} .
$$

\footnotetext{
${ }^{9}$ It has been shown in [23] that in general principal-agent models it is not possible to perfectly screen the market. In most instances there is a non-negligible set of agents who are pushed down to their reservation utilities (bunching of the first type). There is another set where agents stay above their reservation levels, but they choose the same product despite the fact of their different preferences (bunching of the second type). In all likelihood, this behavior is inherited by multi-firm models.
} 
From this point on, given a TBR $f$ we write $f_{1}=f$ and $f_{2}=1-f$. Then $f_{i} \equiv 1$ on $\Theta_{i}, f_{i} \equiv 0$ on $\Theta_{-i}$, and for $\theta_{0} \in \Theta_{0}$ the proportion $f_{i}\left(\theta_{0}\right)$ contracts with firm $i$. We shall rephrase the interaction of the firms in terms of the indirect utilities generated by the catalogues they offer. This allows us to use well established convex analysis machinery. Let

$$
\mathcal{C}_{i} \triangleq\left\{v: \Theta \rightarrow \mathbb{R}_{+} \mid v \geq 0, \exists\{X(\theta)\}_{\theta \in \Theta} \subset \mathcal{X}_{i} \text { s.t. } v^{\prime}(\theta)=-\operatorname{Var}[X(\theta)]\right\} .
$$

These are the sets of all possible (single-firm) indirect utilities that can be generated from incentive compatible catalogues contained in $\mathcal{X}_{i}$. Incentive compatibility is reflected in the requirement $v^{\prime}(\theta)=-\operatorname{Var}[X(\theta)]$, as in Lemma 2.2.

\section{Proposition 2.3 The sets $\mathcal{C}_{i}$ are compact for the topology of uniform convergence.}

\subsection{Some comments on efficiency}

We close this section with some informal comments on efficiency of allocations in our framework. The fundamental theorems of welfare economics establish the equivalence between competitive equilibria (in complete markets) and Pareto-efficient allocations. Agents who trade on a take-it-or-leave-it basis (as in our model) do not respond to given prices in the same way as in a competitive market. As a consequence, the mechanisms that would guarantee (Pareto) efficiency in a Walrasian setting break down. In the sequel we establish the existence of allocations that are efficient in the sense that they maximize aggregate profits, respectively minimize aggregate risk. Specifically, we show that if firms are expected-profit maximizers and if ties are broken in favor of the firm that benefits the most from contracting with the agent, Nash equilibrium allocations are efficient (see Theorem 3.11). It is unclear to us that efficiency can be achieved in a similar fashion if firms minimize risk. This leads us to the notion of socially efficient allocations which we study in Sect. 4. In either case, the presence of a social planer (a regulator) is necessary to achieve efficiency. This indirect market participant plays two important roles: First, he must enforce certain kinds of TBRs in order to guarantee efficient outcomes. Second, he must make sure that individual rationality at the level of firms is preserved. The latter may be achieved by further restricting the choice of admissible TBRs, or by establishing a payment scheme among firms. ${ }^{10}$

\section{Profit maximization}

In this section we analyze a non-cooperative game played among profit-maximizing firms under adverse selection. Our model is an extension to [6]. We show that if efficient tie-breaking rules are implemented (possibly through the influence of a regulator), then the game possesses a Nash equilibrium in mixed strategies.

\subsection{The firms' strategy sets and payoff functions}

The theory of equilibria in multi-firm games developed (among others) in [13,17] and [18] requires the strategy sets to be compact metric spaces. Moreover, the agents' preferences

\footnotetext{
10 An example of a regulated market with a payment scheme is the German health insurance one. For historical reasons some insurers face higher risk customers (e.g. hand laborers) than others. All companies must provide at least a minimal standard of coverage (which is rather high). Furthermore, no potential customer may be turned away. This environment exposes some firms to higher levels of risk. In order to correct the asymmetries in the market, a system of cash-transfers has been set in place.
} 
must be continuous with respect to a topology that makes the strategy sets compact. In other words the strategy sets are generally closed and bounded sets of a finite-dimensional vector space. In the sequel we assume that $\mathcal{X}_{i}$ is the closed convex hull of a finite number of basic products $\left\{X_{i}^{1}, \ldots, X_{i}^{m_{i}}\right\}$. This would be the case, for instance, if firms bundled products and sold fractions to the agents, whose size would not allow them to imitate the firms. We assume that the cost to firm $i$ of delivering $X_{i} \in \mathcal{X}_{i}$ is given by a lower semicontinuous function $K_{i}: \mathcal{X}_{i} \rightarrow \mathbb{R}$. The profit of firm $i$ when it sells claim $X_{i}$ given the price schedule $p_{i}: \mathcal{X}_{i} \rightarrow \mathbb{R}$ is $p_{i}\left(X_{i}\right)-K_{i}\left(X_{i}\right)$. The Competitive Taxation Principle (see [18]) states that the game played over product-price catalogues, i.e. elements of

$$
\mathcal{P}_{i} \triangleq\left\{C_{i} \subset \mathcal{X}_{i} \times P \mid C_{i} \text { closed }\right\}
$$

is as general as the one played over closed subsets of $\mathcal{X}_{i}$ and non-linear price schedules. We recall that $P \subset \mathbb{R}$ is the compact set where feasible prices lie. The firms strategy sets $\mathcal{P}_{i}$ are thus the sets of all compact subsets of $\mathcal{X}_{i} \times P$. We endow $\mathcal{P}_{i}$ with the Hausdorff metric $h$. Since $\mathcal{X}_{i} \times P$ is a compact metric space, so is $\left(\mathcal{P}_{i}, h\right)$ (see, for example [1], Sect. 3.17); furthermore, Tychonoff's theorem guarantees that $\mathcal{P} \triangleq \mathcal{P}_{1} \times \mathcal{P}_{2}$ with the corresponding product metric $h_{p}$ is also compact and metric. We write $\mathcal{B}(\mathcal{P})$ for the Borel $\sigma$-algebra in $\mathcal{P}$. The following proposition allows us to substitute a catalogue profile $\left(C_{1}, C_{2}\right)$ for

$$
\left(\bigcup_{\theta} \mathcal{L}\left(\theta, C_{1}\right), \bigcup_{\theta} \mathcal{L}\left(\theta, C_{2}\right)\right),
$$

as these sets are closed, thus compact:

Proposition 3.1 For any catalogue $C_{i} \in \mathcal{P}_{i}$, the set $\bigcup_{\theta} \mathcal{L}\left(\theta, C_{i}\right)$ is closed.

The maximal attainable profit for firm $i$ from type $\theta$ given the (incentive compatible) catalogue profile $\left(C_{1}, C_{2}\right)$ is

$$
\pi_{i}\left(\theta, C_{1}, C_{2}\right) \triangleq \max \left\{(p-K(X)) \varliminf_{\left\{v_{i} \geq v_{-i}\right\}}(\theta) \mid(X, p) \in C_{i}\right\} .
$$

Here the functions $v_{i}$ are as defined in Sect. 2.4, and $C_{-i}$ enters $\pi_{i}$ via $v_{-i}$. The functions $\pi_{i}$ are the building blocks of the firms' payoff functions.

Proposition 3.2 (Page Jr. [17]) The maps $\pi_{i}: \Theta \times \mathcal{P} \rightarrow \mathbb{R}$ are upper semicontinuous on $\mathcal{P}$ and $\mathcal{B}(\Theta) \times \mathcal{B}(\mathcal{P})$-measurable.

For a given TBR $f$, the payoff function of firm $i$ is given by

$$
\Pi_{i}\left(C_{1}, C_{2}\right) \triangleq \int_{\Theta} \pi_{i}\left(\theta, C_{1}, C_{2}\right) f_{i}(\theta) \mu(d \theta) .
$$

These payoff functions exhibit a highly discontinuous behavior, due to the presence of ties. Only agents who make a positive contribution to a firm's revenues contract with it. This fact plays an important role in the analysis of payoff security contained in Sect. 3.3.2.

\subsection{Efficient tie-breaking rules and reciprocal upper semicontinuity}

In this section we introduce the notion of efficient TBRs:

Definition 3.3 Given a catalogue profile $\left(C_{1}, C_{2}\right)$, a tie-breaking rule $f \in F$ is called efficient if 
- $\pi_{i}\left(\theta, C_{1}, C_{2}\right) \geq \pi_{-i}\left(\theta, C_{1}, C_{2}\right)$ implies $f_{i}(\theta)>0$,

- $\pi_{i}\left(\theta, C_{1}, C_{2}\right)<\pi_{-i}\left(\theta, C_{1}, C_{2}\right)$ implies $f_{i}(\theta)=0$.

The definition above is equivalent to saying that $f^{*}$ is efficient given the catalogue profile $\left(C_{1}, C_{2}\right)$ if and only if

$$
\sum_{i} \Pi_{i}\left(f^{*}, C_{1}, C_{2}\right)=\sup _{f \in F} \sum_{i} \Pi_{i}\left(f, C_{1}, C_{2}\right),
$$

This shows that in general efficient TBRs are endogenously determined.

Definition 3.4 A game $\left\{\left(\Pi_{i}, \mathcal{P}_{i}\right)\right\}$ is said to be reciprocal upper semicontinuous (RUSC) for a given TBR $f$ if the mapping

$$
\left(C_{1}, C_{2}\right) \mapsto \sum_{i} \Pi_{i}\left(f, C_{1}, C_{2}\right)
$$

is upper semicontinuous.

The notion of RUSC games was introduced by Dasgupta and Maskin in [7] (labeled as complementary discontinuous or u.s.c.-sum games) and later generalized by Reny in [22] in order to prove the existence of Nash equilibria in certain discontinuous games. It is shown in [22] that RUSC is inherited by the mixed extension of a game.

Lemma 3.5 (Page Jr. and Monteiro [19]) If the game $\left\{\left(\mathcal{P}_{i}, \Pi_{i}\right)\right\}$ is played using efficient TBRs, then it is RUSC.

Notice that the definition of efficient TBR, together with the linear aggregation of type-wise profits, implies that the mapping $\left(C_{1}, C_{2}\right) \mapsto \sum_{i} \Pi_{i}\left(f, C_{1}, C_{2}\right)$ is independent of the TBR played if the latter it is efficient.

\subsection{Existence of Nash equilibria}

In this section we study necessary conditions for the existence of Nash equilibria in the firms' game. Since the payoff functions $\Pi_{i}$ are not quasiconcave, one cannot prove the existence of pure-strategies equilibria using standard results. Instead we analyze the existence of mixed-strategies equilibria.

\subsubsection{Defining the mixed-strategies game}

Let $\mathcal{M}\left(\mathcal{P}_{j}\right)$ be the set of probability measures on $\left(\mathcal{P}_{i}, \mathcal{B}\left(\mathcal{P}_{i}\right)\right)$. We endow the mixed catalogue strategy sets $\mathcal{M}\left(\mathcal{P}_{i}\right)$ with the $\sigma\left(X^{*}, X\right)$-topology generated by the dual pair $\left\langle\mathbb{L}^{0}\left(\mathcal{P}_{i}\right.\right.$, $\left.\left.\mathcal{B}\left(\mathcal{P}_{i}\right)\right), \mathcal{M}\left(\mathcal{P}_{i}\right)\right\rangle$. The game is played as follows: Each firm chooses an element $\lambda_{i} \in \mathcal{M}\left(\mathcal{P}_{i}\right)$, and its profit under the profile $\lambda=\left(\lambda_{1}, \lambda_{2}\right)$ is given by

$$
\Pi_{i}(\lambda) \triangleq \int_{\mathcal{P}} \Pi_{i}\left(C_{1}, C_{2}\right) \lambda(d C),
$$

where $\lambda(d C)=\lambda_{1}\left(d C_{1}\right) \lambda_{2}\left(d C_{2}\right)$ is the corresponding product measure. By Proposition 3.2 the integrand is measurable, thus expression (2) is well defined. The game $\left\{\left(\mathcal{M}\left(\mathcal{P}_{i}\right), \Pi_{i}\right)\right\}$ is the mixed catalogue game that extends $\left\{\left(\mathcal{P}_{i}, \Pi_{i}\right)\right\}$.

Definition 3.6 A mixed profile $\lambda$ is a Nash equilibrium for the game $\left\{\left(\mathcal{M}\left(\mathcal{P}_{i}\right), \Pi_{i}\right)\right\}$ if

$$
\Pi_{i}\left(\lambda_{i}, \lambda_{-i}\right) \geq \Pi_{i}\left(\lambda_{i}^{\prime}, \lambda_{-i}\right) \quad \text { for all } \quad \lambda_{i}^{\prime} \in \mathcal{M}\left(\mathcal{P}_{i}\right) .
$$

A Nash equilibrium in mixed strategies for $\left\{\left(\mathcal{M}\left(\mathcal{P}_{j}\right), \Pi_{i}\right)\right\}$ can be viewed as an equilibrium in pure strategies for its mixed extension. 


\subsubsection{Uniform payoff security}

A game is said to be compact if the players' strategy sets are compact subsets of a topological space, and their payoff functions are bounded. The mixed extension of the game in hand is a compact game. This is due to the fact that the sets $\mathcal{M}\left(\mathcal{P}_{i}\right)$ are closed, convex and bounded subsets of $\left(\mathbb{L}^{0}\left(\mathcal{P}_{i}, \mathcal{B}\left(\mathcal{P}_{i}\right)\right)\right)^{*}$, thus by the Banach-Alaoglu theorem they are $\sigma\left(X^{*}, X\right)$-compact. Reny provides sufficient conditions for existence of a Nash equilibrium in a compact game in the presence of discontinuous payoff functions. A key requirement is that the game is payoff secure:

Definition 3.7 Given the payoffs $\Pi_{i}: \mathcal{P}_{i} \rightarrow \mathbb{R}$, the catalogue game $\left\{\left(\mathcal{P}_{i}, \Pi_{i}\right)\right\}$ is payoff secure if for all $\left(C_{1}, C_{2}\right) \in \mathcal{P}$, and $\epsilon>0$ there exist $C_{i}^{*} \in \mathcal{P}_{i}$ and $\delta>0$ such that $\Pi_{i}\left(C_{i}^{*}, C_{-i}^{\prime}\right) \geq \Pi_{i}\left(C_{i}, C_{-i}\right)-\epsilon$ for all $C_{-i}^{\prime} \in\left\{D \in \mathcal{P}_{-i} \mid h_{p}\left(D, C_{-i}\right)<\delta\right\}$.

Payoff security of a game does not imply the same property for its mixed extension; however, such is the case with uniform payoff security (see Theorem 1 in [19]). Proving uniform payoff security of a game is simpler than dealing with the weak*-topology to show payoff security of its mixed extension.

Definition 3.8 The game $\left\{\left(\mathcal{P}_{i}, \Pi_{i}\right)\right\}$ is uniformly payoff secure if for all $i=1,2, C_{i} \in \mathcal{P}_{i}$ and $\epsilon>0$ there exist $C_{i}^{*}$ such that for all $C_{-i} \in \mathcal{P}_{-i}$ there exists $\delta>0$ that satisfies $\Pi_{i}\left(C_{i}^{*}, C_{-i}^{\prime}\right) \geq \Pi_{i}\left(C_{i}, C_{-i}\right)-\epsilon$ for all $C_{-i}^{\prime} \in\left\{D \in \mathcal{P}_{-i} \mid h_{p}\left(D, C_{-i}\right)<\delta\right\}$.

Proposition 3.9 The game $\left\{\left(\mathcal{P}_{i}, \Pi_{i}\right)\right\}$ is uniformly payoff secure (hence the game $\left\{\left(\mathcal{M}\left(\mathcal{P}_{j}\right), \Pi_{i}\right)\right\}$ is payoff secure $)$.

Our result on existence of Nash equilibria relies on the following

Theorem 3.10 (Reny [22]) Suppose that $G=\left\{\left(X_{i}, u_{i}\right)\right\}_{i=1}^{m}$ is a compact game which is also quasiconcave, reciprocally upper semicontinuous and payoff secure, then it possesses a pure-strategies Nash equilibrium.

It follows from Lemma 3.5 that the (compact) game $\left\{\left(\mathcal{M}\left(\mathcal{P}_{i}\right), \Pi_{i}\right)\right\}$ is RUSC. The payoff functions are linear in each firm's strategy, hence quasiconcave. Proposition 3.9 then allows us to apply Theorem 3.10 to the mixed extension of the game $\left\{\left(\mathcal{P}_{i}, \Pi_{i}\right)\right\}$ to obtain the following

Theorem 3.11 The game $\left\{\left(\mathcal{P}_{i}, \Pi_{i}\right)\right\}$ possesses a mixed-strategies Nash equilibrium.

\section{Risk minimization}

Consider an insurance market dominated by a few large firms. Each insurer holds a portfolio of risky assets (its customers policies). In order to minimize its risk exposure (assessed in terms of a convex risk measure), each firm lays off parts of its risk on individual agents (via over-the-counter trading of derivatives contracts). In this section we analyze a model for such a risk minimization problem. The model is the multi-firm version of the one introduced in [11]. In contrast with the previous section, we do not seek to prove the existence of Nash equilibria, but rather of socially efficient allocations. The main difficulty towards guaranteeing Nash equilibria stems from the non-linear, per-type impact on the firms' risk assessments. This precludes us from being able to show uniform payoff security and RUSC. 
4.1 The firms' strategy sets and risk assessments

The initial risky endowment of each firm is represented by a random variable $W_{i} \in \mathbb{L}^{2}(\Omega$, $\mathcal{F}, \mathbb{P})$. Firm $i$ assesses its risk exposure using a convex and law invariant risk measure

$$
\varrho_{i}: \mathbb{L}^{2}(\Omega, \mathcal{F}, \mathbb{P}) \rightarrow \mathbb{R} \cup\{+\infty\},
$$

which has the Fatou property. In particular, for any $X \in \mathbb{L}^{2}$ and any $m \in \mathbb{R}, \varrho_{i}(X+m)=$ $\varrho_{i}(X)-m .{ }^{11}$ We refer the reader to [5] and Sect. 4.3 in [9] for detailed discussions on this topic. We restrict the firms' choices to catalogues of the form

$$
C_{i}=\left\{\left(X_{i}(\theta), p_{i}(\theta)\right)\right\}_{\theta \in \Theta} .
$$

This has to be done for technical reasons, thus in this section we focus our attention on inter-firms ties. We are not, however, dealing with a direct revelation mechanism: even if firm 1 were to offer the individually rational and incentive compatible catalogue $C_{1}$, the presence of firm 2 might dissuade agents of type $\theta_{0}$ from choosing $\left(X_{1}\left(\theta_{0}\right), p_{1}\left(\theta_{0}\right)\right)$. For a given catalogue profile $\left(C_{1}, C_{2}\right)$ and a given TBR $f$, the position of firm $i$ after trading is

$$
W_{i}-\int_{\Theta_{i}}\left(p_{i}(\theta)-X_{i}(\theta)\right) \mu(d \theta)-\int_{\Theta_{0}}\left(p_{i}(\theta)-X_{i}(\theta)\right) f_{i}(\theta) \mu(d \theta),
$$

where $\Theta_{i}$ and $\Theta_{0}$ are as defined in Sect. 2.4. From Eq. (1) we have that, if $X(\theta) \in \mathcal{L}\left(\theta, C_{i}\right)$, then form almost all $\theta \in \Theta v_{i}^{\prime}\left(\theta, C_{i}\right)=-\operatorname{Var}[X(\theta)]$. Therefore $v_{i}\left(\theta, C_{i}\right)=E[X(\theta)]+$ $\theta v_{i}^{\prime}\left(\theta, C_{i}\right)-p_{i}(\theta)$. If we rename $X(\theta)=X(\theta)-\mathrm{E}[X(\theta)],{ }^{12}$ then we may write $p_{i}(\theta)=$ $\theta v_{i}^{\prime}\left(\theta, C_{i}\right)-v_{i}\left(\theta, C_{i}\right)$. Firm $i$ 's risk assessment after trading is

$$
A_{i}\left(C_{1}, C_{2}, f\right) \triangleq R_{i}\left(C_{1}, C_{2}, f\right)-I_{i}\left(\left(C_{1}, C_{2}, f\right),\right.
$$

where

$$
R_{i}\left(C_{1}, C_{2}, f\right) \triangleq \varrho_{i}\left(W_{i}-\int_{\Theta_{i}} X_{i}(\theta) \mu(d \theta)-\int_{\Theta_{0}} X_{i}(\theta) f_{i}(\theta) \mu(d \theta)\right),
$$

denotes firm $i$ 's risk. The corresponding income is given by

$$
I_{i}\left(( C _ { 1 } , C _ { 2 } , f ) \triangleq \int _ { \Theta _ { i } } \left(\theta v_{i}^{\prime}(\theta)-v_{i}(\theta) \mu(d \theta)+\int_{\Theta_{0}}\left(\theta v_{i}^{\prime}(\theta)-v_{i}(\theta)\right) f_{i}(\theta) \mu(d \theta) .\right.\right.
$$

When a catalogue profile $\left(C_{1}, C_{2}\right)$ is offered, the corresponding indirect utility functions $\left(v_{1}, v_{2}\right)$ show how the market is segmented. The more interesting set is $\Theta_{0}$, i.e. the set of indifferent agents. Within $\Theta_{0}$, there are two intrinsically different situations. First, $v_{1}$ and $v_{2}$ may be identical over a non-negligible subset. These could be regarded as "true" ties, in the sense that they will have different impacts on the firms' risk assessments under different TBRs. Second, there could be types for which $v_{1}$ and $v_{2}$ are secant (see Definition 4.1 below). We show below that given our assumptions on $\mu$, these types do not have a direct impact on the aggregate incomes. They do, however, indicate the points where agents switch from contracting with one firm to the other. In other words they show where the customers' affiliations shift.

\footnotetext{
11 This property is known as translation invariance. We require it repeatedly below.

12 This is possible due to the translation invariance of $\varrho_{i}$, and fully characterizes $p_{i}$ in terms of $v_{i}$.
} 
Definition 4.1 Given a pricing schedule $\left(v_{1}, v_{2}\right) \in \mathcal{C}_{1} \times \mathcal{C}_{2}$, we say that there is a shift in the customers' affiliations at $\theta \in \operatorname{int}(\Theta)$ if

$$
\left(v_{1}\left(\theta_{-}\right)-v_{2}\left(\theta_{-}\right)\right) \cdot\left(v_{1}\left(\theta_{+}\right)-v_{2}\left(\theta_{+}\right)\right)<0 .
$$

Proposition 4.2 For any pricing schedule $\left(v_{1}, v_{2}\right) \in \mathcal{C}_{1} \times \mathcal{C}_{2}$ the set $\Theta_{s}$ has at most countably many elements. Furthermore, the derived set of $\Theta_{s}$ has measure zero.

As a consequence of the Proposition 4.2, the set of pre-images of the crossings of the graphs of two functions $v_{1} \in \mathcal{C}_{i}$ and $v_{2} \in \mathcal{C}_{2}$ has $\mu$-measure zero, which yields the following

Corollary 4.3 Let $v_{1} \in \mathcal{C}_{1}$ and $v_{2} \in \mathcal{C}_{2}$, then the set $\left\{v_{1}=v_{2}\right\} \cap\left\{v_{1}^{\prime} \neq v_{2}^{\prime}\right\}$ is of $\mu$-measure zero.

The aggregate income of the firms is then independent of the TBR, namely

$$
\sum_{i} I_{i}\left(C_{1}, C_{2}, f\right)=\int_{\Theta_{0}}\left(\theta v(\theta)-v^{\prime}(\theta)\right) \mu(d \theta)+\sum_{i} \int_{\Theta_{i}}\left(\theta v_{i}^{\prime}(\theta)-v_{i}(\theta) \mu(d \theta) .\right.
$$

Finally we define

$$
\mathrm{A}\left(C_{1}, C_{2}, f\right) \triangleq \sum_{i} A_{i}\left(C_{1}, C_{2}, f\right)
$$

\subsection{Socially efficient allocations}

A market situation $\left(C_{1}^{*}, C_{2}^{*}\right)$, together with a TBR $f^{*}$ is said to be a socially efficient allocation (henceforth SEA) if it minimizes the aggregate risk in the economy and if it is individually rational at the firms' level; in other words if $\mathrm{A}\left(C_{1}^{*}, C_{2}^{*}, f^{*}\right)$ solves the problem

$$
\Psi \triangleq \inf _{v} \inf _{f} \inf _{X}\left\{\mathrm{~A}\left(C_{1}, C_{2}, f\right) \mid-\operatorname{Var}\left[X_{i}(\theta)\right]=v_{i}^{\prime}(\theta), A_{i}\left(C_{1}, C_{2}, f\right) \leq \varrho_{i}\left(W_{i}\right)\right\} .
$$

As it was the case in Lemma 2.2, the variance constraints $-\operatorname{Var}\left[X_{i}(\theta)\right]=v_{i}^{\prime}(\theta)$ capture the incentive compatibility of the catalogues $C_{i}$. The constraint $A_{i}\left(C_{1}, C_{2}, f\right) \leq \varrho_{i}\left(W_{i}\right)$ represents firm $i$ 's individual rationality. In absence of a competitive market one cannot rely on equilibrium pricing to take care of the issue of individual rationality. An alternative would be to establish a cash-transfer system, which should be supervised by the regulator. We comment further on the latter in Sect. 4.2.4. In the remainder of this section we study the existence of SEAs, and we show that the presence a regulator is required in order for such allocations to be attainable and/or implementable. Our existence result depends heavily on the implementation of efficient TBRs.

Definition 4.4 Let $C=\left(C_{1}, C_{2}\right)$ be a catalogue profile. A tie-breaking rule $\bar{f} \in F$ is efficient for $C$ if

$$
\mathrm{A}\left(C_{1}, C_{2}, \bar{f}\right)=\inf _{f \in F}\left\{\mathrm{~A}\left(C_{1}, C_{2}, f\right)\right\}
$$

From the definition above one observes that efficient TBRs are endogenously determined. Here we encounter the first need for the social planner in our risk-minimization setting. It could be the case that unless regulated, efficient TBRs would not be implemented. 


\subsubsection{Minimizing the risk for fixed incomes and tie-breaking rule}

In a first step, we fix $\left(v_{1}, v_{2}\right) \in \mathcal{C}_{1} \times \mathcal{C}_{2}$ (hence the firms' incomes), as well as $f \in F$. We shall abuse notation slightly and write $R_{i}\left(v_{1}, v_{2}, X_{i}, f\right)$ for $R_{i}\left(C_{1}, C_{2}, f\right)$. We then analyze the problem

$$
\Psi_{1} \triangleq \inf _{\left(X_{1}, X_{2}\right)}\left\{\sum_{i} R_{i}\left(v_{1}, v_{2}, X_{i}, f\right) \mid-\operatorname{Var}\left[X_{i}(\theta)\right]=v_{i}^{\prime}(\theta) \text { and } A_{i}\left(v_{1}, v_{2}, X_{i}, f\right) \leq \varrho_{i}\left(W_{i}\right)\right\} .
$$

This problem can be decoupled into the sum of the infima, since the choice of $X_{i}$ bears no weight on the evaluation of $R_{-i}$. We study the solution(s) to the following single-firm problems:

$$
\inf _{X_{i}}\left\{R_{i}\left(v_{i}, v_{-i}, X_{i}, f\right) \mid-\operatorname{Var}\left[X_{i}(\theta)\right]=v_{i}^{\prime}(\theta) \text { and } A_{i}\left(v_{1}, v_{2}, X_{i}, f\right) \leq \varrho_{i}\left(W_{i}\right)\right\} .
$$

To deal with the individual rationality constraints, we define

$$
\mathcal{X}_{i}^{v, f} \triangleq\left\{X_{i} \in \mathcal{X}_{i} \mid A_{i}\left(v_{1}, v_{2}, X_{i}, f\right) \leq \varrho_{i}\left(W_{i}\right)\right\},
$$

which is the set of individually rational products for the given price schedule $\left(v_{1}, v_{2}\right)$, and

$$
\mathcal{X}_{i}^{k_{i}^{v}} \triangleq\left\{X \in \mathcal{X}_{i} \mid\left\|X_{i}\right\|_{2}^{2} \leq k_{i}^{v}\right\}
$$

where $k_{i}^{v} \triangleq v_{i}(a)-v_{i}(0)$. The set $\mathcal{X}_{i}^{k_{i}^{v}}$ contains all the products that can be structured as to construct incentive compatible catalogues given $\left(v_{1}, v_{2}\right)$. The quantity $k_{i}^{v}$ provides a bound to the $\mathbb{L}^{2}$-norm of the products via the constraint $-\operatorname{Var}\left[X_{i}(\theta)\right]=v_{i}^{\prime}(\theta)$. We elaborate further into the $k_{i}^{v}$ 's in Appendix B, where we provide an outline of the proof of Lemma 4.5. The proof is analogous to that of Theorem 2.3 in [11], and we include it for completeness.

Lemma 4.5 For $v \in \mathcal{C}_{1} \times \mathcal{C}_{2}$ and $f \in F$ given, if $\mathcal{X}_{i}^{k_{i}^{v}} \cap \mathcal{X}_{i}^{v, f} \neq \emptyset$ then there exist $X_{i}^{v, f} \in \mathcal{X}_{i}$ such that

$$
\Psi_{1}=R_{1}\left(v_{1}, v_{2}, X_{1}^{v, f}, f\right)+R_{2}\left(v_{1}, v_{2}, X_{2}^{v, f}, f\right) .
$$

In order to establish the existence of an efficient TBR it is important to characterize the optimal contracts $X_{i}^{v, f}$. We show below that $X_{i}^{v, f}$ can be decomposed into a $\theta$-dependent function and an $\omega$-dependent random variable. To this end, we construct the Lagrangian associated to minimizing $R_{i}\left(v_{i}, v_{-i}, X_{i}, f\right)$ subject to the moment conditions $\mathrm{E}\left[X_{i}(\theta)\right]=0$ and $\operatorname{Var}\left[X_{i}(\theta)\right]+v_{i}^{\prime}(\theta)=0$, and we compute the Frechét differential of $R_{i}$ at $X_{i}$ in the direction of $h \in \mathcal{X}_{i}:$

$$
R_{i}^{\prime}\left(X_{i}\right) h=\varrho_{i}^{\prime}\left(W_{i}-\int_{\Theta_{i}} X_{i}(\theta) \mu(d \theta)-\int_{\Theta_{0}} X_{i}(\theta) f_{i}(\theta) \mu(d \theta)\right)\left(-\int_{\Theta_{i}} h(\theta) d \theta-\int_{\Theta_{0}} h(\theta) f_{i}(\theta) \mu(d \theta)\right) .
$$

Since, for all $H \in L^{2}(\Omega, \mathbb{P})$, the map $K \mapsto \varrho_{i}^{\prime}(H) K$ is linear, it follows from the Riesz representation theorem that there is a random variable $Z_{X_{i}^{v, f}} \in L^{2}(\Omega, \mathbb{P})$ such that

$$
R_{i}^{\prime}\left(X_{i}\right) h=\int_{\Omega} Z_{X_{i}^{v, f}}\left(-\int_{\Theta_{i}} h(\theta) d \theta-\int_{\Theta_{0}} h(\theta) f_{i}(\theta) \mu(d \theta)\right) d \mathbb{P} .
$$

Let $g: F \rightarrow \mathbb{L}^{0}(\Theta, \mu)$ be given by

$$
g(f)=1 \mathrm{l}_{\Theta_{i}}+1 \mathrm{l}_{\Theta_{0}} f .
$$


The operator $R_{i}$ has an extremum at $X_{i}$ under our moment constraints if there exist Lagrange multipliers $\lambda_{i}, \eta_{i} \in \mathbb{L}^{2}(\Theta, \mu)$ such that

$$
\int_{\Omega} \int_{\Theta} h(\theta)\left(-Z_{X_{i}^{v, f}} g\left(f_{i}\right)(\theta)+\eta_{i}(\theta)+2 \lambda_{i}(\theta) X_{i}(\theta)\right) \mu(d \theta) d \mathbb{P}=0
$$

for all $h \in \mathcal{X}_{i}$. Since $(\theta, \omega) \rightarrow-Z_{X_{i}^{v, f}} g\left(f_{i}\right)(\theta)+\eta_{i}(\theta)+2 \lambda_{i}(\theta) X_{i}(\theta)$ is an integrable function, the DuBois-Reymond lemma implies

$$
-Z_{X_{i}^{v, f}} g\left(f_{i}\right)(\theta)+\eta_{i}(\theta)+2 \lambda_{i}(\theta) X_{i}(\theta)=0 .
$$

Using the moment conditions $\mathrm{E}_{\mathbb{P}}\left[X_{i}^{v, f}(\theta)\right]=0$ and $\operatorname{Var}\left[X_{i}^{v, f}(\theta)\right]=-v_{i}^{\prime}(\theta)$ we obtain

$$
\eta_{i}(\theta)=\mathrm{E}_{\mathbb{P}}\left[Z_{X_{i}^{v, f}}\right] g\left(f_{i}\right)(\theta) \text { and } \lambda_{i}(\theta)=\frac{g\left(f_{i}\right)(\theta) \sqrt{\operatorname{Var}\left[Z_{X_{i}^{v, f}}\right]}}{2 \sqrt{-v_{i}^{\prime}(\theta)}} .
$$

Inserting the expressions for the Lagrange multipliers into Eq. (3) yields

$$
X_{i}^{v, f}(\theta)=\sqrt{-v_{i}^{\prime}(\theta)} \bar{Z}_{i}^{v, f},
$$

where

$$
\bar{Z}_{i}^{v, f} \triangleq\left(Z_{X_{i}^{v, f}}-\mathrm{E}\left[Z_{X_{i}^{v, f}}\right]\right) / \sqrt{\operatorname{Var}\left[Z_{X_{i}^{v, f}}\right]}
$$

Equation (4) shows that the minimizers of problem $\Psi_{1}$ form collinear families in $\mathcal{X}$. Moreover, the randomness stemming from $(\Omega, \mathbb{P})$ and the one induced by $(\Theta, \mu)$ are decoupled. This property will prove to be key in Sect. 4.2.2. The previous discussion yields the following characterization result:

Proposition 4.6 Let $\left(X_{1}^{v, f}, X_{2}^{v, f}\right)$ be a solution to $\Psi_{1}$. Then $X_{i}^{v, f}$ takes the form

$$
X_{i}^{v, f}(\theta, \omega)=\sqrt{-v_{i}^{\prime}(\theta)} \bar{Z}_{i}^{v, f}(\omega)
$$

for some normalized random variable $\bar{Z}_{i}^{v, f}$ on $(\Omega, \mathcal{F}, \mathbb{P})$.

If either $X_{1}^{v, f}$ or $X_{2}^{v, f}$ do not satisfy $A_{i}\left(v_{1}, v_{2}, X_{i}^{v, f}, f\right) \leq \varrho_{i}\left(W_{i}\right)$, then using the standard convention $\inf \{\emptyset\}=\infty$ we would have $\Psi_{1}=\infty$. In terms of the program $\Psi$, this guarantees that only pricing schedules and TBRs that offer the possibility of constructing individually rational catalogues stand a chance to be chosen.

\subsubsection{Existence of efficient tie-breaking rules}

In this section we show that for a given price schedule $\left(v_{1}, v_{2}\right)$ there is a TBR $f^{v}$ such that $\left(X_{1}^{v, f^{v}}, X_{2}^{v, f^{v}}\right)$ minimize the aggregate risk evaluation of the firms. For $v=\left(v_{1}, v_{2}\right) \in \mathcal{C}_{1} \times \mathcal{C}_{2}$ we define

$$
\tilde{R}_{i}\left(v_{1}, v_{2}, f\right) \triangleq \varrho_{i}\left(W_{i}-Z_{i}^{v, f}(\omega)\left(\int_{\Theta_{i}} \sqrt{-v_{i}^{\prime}(\theta)} d \theta+\int_{\Theta_{0}} f_{i}(\theta) \sqrt{-v_{i}^{\prime}(\theta)} \mu(d \theta)\right)\right)
$$

and

$$
F_{v} \triangleq\left\{f \in F \mid \sum_{i} \tilde{R}_{i}\left(v_{1}, v_{2}, f\right)<\infty\right\}
$$


We have to solve the problem

$$
\Psi_{2} \triangleq \inf _{F_{v}} \sum_{i} \tilde{R}_{i}\left(v_{1}, v_{2}, f\right)
$$

If $F_{v}=\emptyset$, then as above $\Psi_{2}=\infty$. Otherwise we must verify the lower semicontinuity of the mapping

$$
f \mapsto \tilde{R}_{1}\left(v_{1}, v_{2}, f\right)+\tilde{R}_{2}\left(v_{1}, v_{2}, f\right), \quad f \in F_{v} .
$$

To this end consider a minimizing sequence $\left\{f^{n}\right\} \subset F_{v}$. The Banach-Alaoglu theorem guarantees that $\left\{f^{n}\right\}$ is weakly convergent ${ }^{13}$ to some $f^{v} \in F_{v}$. Since $V_{i}(\theta) \triangleq \sqrt{-v_{i}^{\prime}(\theta)}$ belongs to $\mathbb{L}^{2}(\Theta, \mu)$ for all $v_{i} \in \mathcal{C}_{i}$, then

$$
\int_{\Theta_{0}} f_{i}^{n}(\theta) V_{i}(\theta) d \theta \rightarrow \int_{\Theta_{0}} f_{i}^{v}(\theta) V_{i}(\theta) \mu(d \theta) .
$$

Let

$$
a_{i}^{v, n} \triangleq \int_{\Theta_{i}} V_{i}(\theta) d \theta+\int_{\Theta_{0}} f_{i}^{n}(\theta) V_{i}(\theta) \mu(d \theta)
$$

For $f^{v}$ we define $a_{i}^{v}$ analogously. From (5) we have that $a_{i}^{v, n} \rightarrow a_{i}^{v}$. Since $\left\|Z_{i}^{v, f^{n}}\right\|_{2} \leq 1$ for all $n$, there exists $Z_{i}^{v} \in \mathbb{L}^{2}(\Omega, \mathbb{P})$ such that $\left\|Z_{i}^{v}\right\|_{2} \leq 1$ and

$$
Z_{i}^{v, f^{n}} \stackrel{w}{\rightarrow} Z_{i}^{v} \quad \text { (weak convergence). }
$$

Therefore $Y_{i}^{v, n} \triangleq a_{i}^{v, n} \cdot Z_{i}^{v, f^{n}}$ converges weakly to $Y_{n}^{v} \triangleq a_{i}^{v} \cdot Z_{i}^{v}$. It follows from the Fatou property of $\varrho_{i}$ that

$$
\varrho_{i}\left(W_{i}-a_{i}^{v} \cdot Z_{i}^{v}\right) \leq \liminf _{n \rightarrow \infty} \varrho_{i}\left(W_{i}-a_{i}^{v, n} \cdot Z_{i}^{v, f^{n}}\right) .
$$

From the lower semicontinuity of the norm in terms of weak convergence we have

$$
\operatorname{Var}\left[Z_{i}^{v} V_{i}(\theta)\right] \leq-v_{i}^{\prime}(\theta) .
$$

Proceeding as in Sect. 4.2.1, we have that

$$
\tilde{R}_{i}\left(v_{1}, v_{2}, f^{v}\right) \leq \varrho_{i}\left(W_{i}-a_{i}^{v} \cdot Z_{i}^{v}\right)
$$

Therefore

$$
\sum_{i=1}^{2}\left(\tilde{R}_{i}\left(v_{1}, v_{2}, f^{v}\right)-I_{i}\left(v_{1}, v_{2}, f^{v}\right)\right)=\inf _{f \in F}\left\{\sum_{i=1}^{2}\left(\tilde{R}_{i}\left(v_{1}, v_{2}, f\right)-I_{i}\left(v_{1}, v_{2}, f\right)\right)\right\} .
$$

We denote by $\left(X_{1}^{v}, X_{2}^{v}\right)$ any optimal list of claims associated to the pricing schedules $\left(v_{1}, v_{2}\right)$ and the TBR $f^{v}$. For notational convenience we define

$$
\mathrm{A}\left(v_{1}, v_{2}\right) \triangleq \sum_{i=1}^{2}\left(\tilde{R}_{i}\left(v_{1}, v_{2}, f^{v}\right)-I_{i}\left(v_{1}, v_{2}, f^{v}\right)\right)
$$

\footnotetext{
13 We omit writing "up to a subsequence" when exploiting the compactness of sets, but it should of course be kept in mind.
} 


\subsubsection{Minimizing with respect to the firms' incomes}

To finalize the proof of existence of a SEA, we let $\left\{\left(v_{1}^{n}, v_{2}^{n}\right)\right\}$ be a minimizing sequence of

$$
\sum_{i=1}^{2}\left(\tilde{R}_{i}\left(v_{1}, v_{2}, f^{v}\right)-I_{i}\left(v_{1}, v_{2}, f^{v}\right)\right) .
$$

We get from Proposition 2.3 that there exist $\left(\bar{v}_{1}, \bar{v}_{2}\right) \in \mathcal{C}_{1} \times \mathcal{C}_{2}$ such that $v_{i}^{n} \rightarrow \bar{v}_{i}$ uniformly. This implies that $V_{i}^{n} \rightarrow \bar{V}_{i}$ almost surely (see for example Proposition A.4 in [8]). Moreover, for any $\theta \in \Theta$ where convergence holds, it is uniform. We have from Fatou's lemma and the fact that $0 \leq-I_{i}\left(v_{1}, v_{2}, f\right)$ for any $\left(v_{1}, v_{2}\right) \in \mathcal{C}_{1} \times \mathcal{C}_{2}$ and any $f \in F$ that

$$
\sum_{i=1}^{2}-I_{i}\left(\bar{v}_{1}, \bar{v}_{2}, f^{\bar{v}}\right) \leq \liminf _{n \rightarrow \infty} \sum_{i=1}^{2}-I_{i}\left(v_{1}^{n}, v_{2}^{n}, f^{v^{n}}\right) .
$$

Let $\langle\cdot, \cdot\rangle$ be the canonical inner product in $\mathbb{L}^{2}(\Omega, \mathbb{P})$. In order to deal with the risky part of the firms' problems we require the following

Lemma 4.7 Let $\left\{\phi_{n}\right\},\left\{\psi_{n}\right\} \subset \mathbb{L}^{2}(\Omega, \mathbb{P})$ and $\phi, \psi \in \mathbb{L}^{2}(\Omega, \mathbb{P})$ be such that

$$
\phi_{n} \stackrel{\|\cdot\|_{2}}{\longrightarrow} \phi \text { and } \psi_{n} \stackrel{w}{\rightarrow} \psi, \text { then }\left\langle\phi_{n}, \psi_{n}\right\rangle \rightarrow\langle\phi, \psi\rangle .
$$

We show in Proposition 4.8 that if it the market segments exhibited no jumps in their limiting behavior, then there would exist SEAs. By absence of jumps we mean that

$$
11_{\Theta_{0}^{n}} \stackrel{a . s .}{\longrightarrow} 1_{\bar{\Theta}_{0}} \text { and } 11_{\Theta_{i}^{n}} \stackrel{\text { a.s. }}{\longrightarrow} 1_{\bar{\Theta}_{i}},
$$

where $\bar{\Theta}_{0}=\left\{\bar{v}_{1}=\bar{v}_{2}\right\}$ and $\bar{\Theta}_{i}=\left\{\bar{v}_{i}>\bar{v}_{-i}\right\}$. This "nice" convergence of the $\Theta_{i}^{n}$ 's is by no means the general scenario. As an example let $v_{1}^{n} \equiv v_{1}$ and $v_{2}^{n}=v_{1}+1 / n$. Here $\Theta_{1}^{n} \equiv \Theta, \Theta_{2} \equiv \emptyset$, but in the limit only $\bar{\Theta}_{0} \neq \emptyset$. Nonetheless, the existence result of SEAs under the assumption of convergence of the indicator functions is relevant for the general case, and we present it below.

Proposition 4.8 Let $\left\{\left(v_{1}^{n}, v_{2}^{n}\right)\right\},\left(\bar{v}_{1}, \bar{v}_{2}\right) \subset \mathcal{C}_{1} \times \mathcal{C}_{2}$ such that $v_{i}^{n} \rightarrow \bar{v}_{i}$ uniformly. Assume that $\mathrm{l}_{\Theta_{j}^{n}} \rightarrow 11_{\bar{\Theta}_{j}}$, and let $\bar{X}_{1}, \bar{X}_{2}$ and $\bar{f}$ be the (weak) limits of $X_{1}^{v^{n}}, X_{2}^{v^{n}}$ and $f^{v^{n}}$. Then

$$
\begin{aligned}
\liminf _{n \rightarrow \infty} \sum_{i=1}^{2} \tilde{R}_{i}\left(v_{1}^{n}, v_{2}^{n}, f^{v^{n}}\right) & \geq \sum_{i=1}^{2} \varrho_{i}\left(W_{i}-\int_{\bar{\Theta}_{i}} \bar{X}_{i} \mu(d \theta)-\int_{\bar{\Theta}_{0}} \bar{f}_{i} \bar{X}_{i} \mu(d \theta)\right) \\
& \geq \sum_{i=1}^{2} \varrho_{i}\left(W_{i}-\int_{\bar{\Theta}_{i}} X_{i}^{\bar{v}} \mu(d \theta)-\int_{\bar{\Theta}_{0}} f_{i}^{\bar{v}} X_{i}^{\bar{v}} \mu(d \theta)\right),
\end{aligned}
$$

where $\left(X_{1}^{\bar{v}}, X_{2}^{\bar{v}}, f^{\bar{v}}\right)$ solves the social planer's problem for $\left(\bar{v}_{1}, \bar{v}_{2}\right)$.

We now deal with the possibility of non-convergence of the indicator functions. We first observe that the $\Theta_{0}^{n}$, s are closed subsets of $\Theta$, which is compact. The set

$$
\overline{2}^{\Theta}:=\{A \subset \Theta \mid A \neq \emptyset \text { and } A \text { is closed }\}
$$

endowed with the Hausdorff metric $h: \overline{2}^{\Theta} \rightarrow \mathbb{R}_{+}$is a compact metric space (see for example [1], Chapter 3). If $\Theta_{0}^{n} \neq \emptyset$ infinitely often, then there exists $\hat{\Theta}_{0} \in \overline{2}^{\Theta}$ such that $\Theta_{0}^{n} \stackrel{h}{\rightarrow} \hat{\Theta}_{0}$. 
Moreover, $\hat{\Theta}_{0}$ is contained in $\bar{\Theta}_{0}$. If, on the contrary, $\Theta_{0}^{n}=\emptyset$ for all but a finite number of $n$ 's, then we define the Hausdorff limit of $\left\{\Theta_{0}^{n}\right\}$ as the empty set, which is again contained in $\bar{\Theta}_{0}$. In both instances we observe that there can only be more ties in the limit, and hence more ways of breaking them. This suggests that the aggregate risk in the limit is indeed no greater than the aggregate risk in the pre-limit.

As for the sets $\Theta_{i}^{n}$, if $\theta$ is not eventually in either of them, then either $\theta \in \hat{\Theta}_{0}$ or it is a type whose affiliation alternates. However, the limiting behavior of the latter is that of indifference, due to the convergence of the functions $v_{i}^{n}$. In other words, an agent type $\theta$ eventually always contracts with the same firm $i$, alternates between firms or is always indifferent. Thus, $\theta \in \Theta_{0}^{n}$ for all sufficiently large n or $\theta \in \bar{\Theta}_{0}$. We now decompose the type space into subsets for which the associated indicator functions converge. We define

$$
\tilde{\Theta}_{0}^{n} \triangleq \bar{\Theta}_{0} \backslash \Theta_{0}^{n}, \quad \text { and } \quad \tilde{\Theta}_{i}^{n} \triangleq \Theta_{i}^{n} \backslash \tilde{\Theta}_{0}^{n} .
$$

The sets $\tilde{\Theta}_{i}^{n}$ contain the "surviving customers", in the sense that there will be no jumps towards indifference from types in $\tilde{\Theta}_{i}^{n}$ at the limit; $\tilde{\Theta}_{0}^{n}$ is the set of "alternating customers". By construction

$$
\Theta=\tilde{\Theta}_{0}^{n} \cup \Theta_{0}^{n} \cup \tilde{\Theta}_{1}^{n} \cup \tilde{\Theta}_{2}^{n}=\bar{\Theta}_{0} \cup \tilde{\Theta}_{1}^{n} \cup \tilde{\Theta}_{2}^{n} .
$$

The following lemma shows that the sets $\tilde{\Theta}_{i}^{n}$ converge to the sets of agent types that contract with firm $i$ when $\left(\bar{v}_{1}, \bar{v}_{2}\right)$ is offered.

Lemma 4.9 For $i=1,2$ we have $1_{\tilde{\Theta}_{i}^{n}} \rightarrow 11_{\bar{\Theta}_{i}}$.

Let us now assume that the social planer's problem were such that, at every stage $n$, all agent types that belong to the set of "alternating customers" are deemed indifferent. This results in more tie-breaking possibilities. Specifically we consider the social planer's problem

$\mathrm{A}^{*}\left(v_{1}^{n}, v_{2}^{n}\right)=\inf _{f^{n} \in F} \inf _{X}\left\{\sum_{i=1}^{2} \varrho_{i}\left(W_{i}-\int_{\tilde{\Theta}_{i}^{n}} X_{i}^{n} \mu(d \theta)-\int_{\tilde{\Theta}_{0}^{n} \cup \Theta_{0}^{n}} f_{i}^{n} X_{i}^{n} \mu(d \theta)\right) \mid \operatorname{Var}\left[X_{i}^{n}(\theta)\right]=-\left(v_{i}^{n}\right)^{\prime}(\theta)\right\}$.

A subset of the possible choices of the $f_{i}^{n}$ 's is $F_{0}^{n}$, which is defined as the set of $\tilde{f} \in F$ such that

$$
\tilde{f}(\theta)= \begin{cases}0, & v_{i}^{n}(\theta)<v_{-i}^{n}(\theta) \\ 1, & v_{i}^{n}(\theta)>v_{-i}^{n}(\theta) \\ f(\theta), & \text { otherwise }\end{cases}
$$

for some TBR $f \in F$. We then have that $\left.\mathrm{A}^{*}\left(v_{1}^{n}, v_{2}^{n}\right)\right|_{F_{0}}=\mathrm{A}\left(v_{1}^{n}, v_{2}^{n}\right)$. Thus, $\mathrm{A}\left(v_{1}^{n}, v_{2}^{n}\right) \geq$ $\mathrm{A}^{*}\left(v_{1}^{n}, v_{2}^{n}\right)$. Consider a minimizing sequence $\left\{\left(v_{1}^{n}, v_{2}^{n}\right)\right\}$ with (uniform) limit $\left(\bar{v}_{1}, \bar{v}_{2}\right)$. Lemma 4.9 guarantees that $11_{\tilde{\Theta}_{i}^{n}} \rightarrow 1_{\bar{\Theta}_{i}}$; moreover $\tilde{\Theta}_{0}^{n} \cup \Theta_{0}^{n} \equiv \bar{\Theta}_{0}$ so patently $11_{\tilde{\Theta}_{0}^{n} \cup \Theta_{0}^{n}} \rightarrow 11_{\bar{\Theta}_{0}}$. These two facts allow us to apply Proposition 4.8 to $\mathrm{A}^{*}\left(v_{1}^{n}, v_{2}^{n}\right)$, which yields

$$
\begin{aligned}
\liminf _{n \rightarrow \infty} \mathrm{A}\left(v_{1}^{n}, v_{2}^{n}\right) & \geq \liminf _{n \rightarrow \infty} \mathrm{A}^{*}\left(v_{1}^{n}, v_{2}^{n}\right) \\
& \geq \sum_{i=1}^{2} \varrho_{i}\left(W_{i}-\int_{\bar{\Theta}_{i}} X_{i}^{*} \mu(d \theta)-\int_{\bar{\Theta}_{0}} f_{i}^{*} X_{i}^{*} \mu(d \theta)\right),
\end{aligned}
$$

where $\left(X_{1}^{*}, X_{2}^{*}, f^{*}\right)$ solves the social planer's problem for $\left(\bar{v}_{1}, \bar{v}_{2}\right)$. This shows that $\left(X_{1}^{*}, X_{2}^{*}\right.$, $\left.f^{*}\right)$ is indeed optimal because $\left\{\left(v_{1}^{n}, v_{2}^{n}\right)\right\}$ was required to be a minimizing sequence. We have proved the following 
Theorem 4.10 If firm $i$ assesses risk using a law invariant risk measure $\varrho_{i}: \mathcal{X} \rightarrow$ $\mathbb{R} \cup\{+\infty\}$, which has the Fatou property, and if it offers catalogues of the form $C_{i}=$ $\left\{\left(X_{i}(\theta), p_{i}(\theta)\right)\right\}_{\theta \in \Theta}$, then there exists a socially efficient market situation.

\subsubsection{Individual rationality revisited}

Assume that $\left(C_{1}^{*}, C_{2}^{*}, f^{*}\right)$ is a solution to the problem $\Psi$ without the individual rationality constraints $\hat{R}_{i}\left(C_{1}, C_{2}, f\right) \leq \varrho_{i}\left(W_{i}\right)$. Since both firms could simply offer $(0,0)$, a minimization of $\mathrm{A}(\cdot, \cdot, \cdot)$ would be IR on the aggregate level. Then there would exist $r \geq 0$ (the rent of risk exchange) such that

$$
A\left(C_{1}^{*}, C_{2}^{*}, f^{*}\right)=\varrho_{1}\left(W_{1}\right)+\varrho_{2}\left(W_{2}\right)-r
$$

In this setting, the regulator would have enforce the cash-transfer. We define a transfer $S E A$ to be a quadruple $\left(C_{1}^{*}, C_{2}^{*}, f^{*}, T^{*}\right)$ such that

$\Psi=A\left(C_{1}^{*}, C_{2}^{*}, f^{*}\right) ; \hat{R}_{1}\left(C_{1}^{*}, C_{2}^{*}, f^{*}\right)-T^{*} \leq \varrho_{1}\left(W_{1}\right) ; \hat{R}_{2}\left(C_{1}^{*}, C_{2}^{*}, f^{*}\right)+T^{*} \leq \varrho_{2}\left(W_{2}\right) ;$ and $T^{*} \in\left[r_{1}, r_{2}\right]$ for some $r_{1}, r_{2} \in \mathbb{R}$ such that $r_{2}-r_{1}=r$. The arguments contained in Sects. 4.2.1, 4.2.2 and 4.2.3 can be immediately applied to prove the following

Corollary 4.11 If firm $i$ assesses risk using a law invariant risk measure $\varrho_{i}: \mathcal{X} \rightarrow$ $\mathbb{R} \cup\{+\infty\}$, which has the Fatou property, and if it offers catalogues of the form $C_{i}=$ $\left\{\left(X_{i}(\theta), p_{i}(\theta)\right)\right\}_{\theta \in \Theta}$, then there exists a transfer socially efficient market situation.

\section{Examples: risk Minimization}

In this section we focus our attention on two well-known risk measures: average value at risk and entropic. For the entropic risk measure, we show there is a SEA where both firms service all of the agents. In this case the optimal TBR is a constant proportion over the whole market. We refer to such efficient TBRs as "fix-mix" rules.

\subsection{Entropic-risk-minimizing firms}

In what follows we assume firms use the entropic risk measure to assess their risk exposure, i.e.

$$
\varrho_{i}(X)=\frac{1}{\gamma_{i}} \ln \mathrm{E}_{\mathbb{P}}\left[\exp \left(-\gamma_{i} X\right)\right]
$$

The coefficient $\gamma_{i}$ represents firm $i$ 's risk aversion. This particular choice of risk measures, which is closely related to exponential utility, allows us to further the analysis into the structure of SEAs. For $v_{i}$ and $f$ given, Proposition 4.6 allows us to write program $\Psi_{1}$ (the minimization with respect to the firms' claims for fixed incomes and TBR in Sect. 4.2.1) as

$$
\inf _{\left(Z_{1}, Z_{2}\right) \in \Gamma_{i} \times \Gamma_{2}} \sum_{i=1}^{2} \varrho\left(W-Z_{i}\left(\int_{\Theta_{i}} \sqrt{-v_{i}^{\prime}(\theta)} \mu(d \theta)+\int_{\Theta_{0}} \sqrt{-v_{i}^{\prime}(\theta)} f_{i}(\theta) \mu(d \theta)\right)\right),
$$

where

$$
\Gamma_{i}:=\left\{Z \in \mathcal{X}_{i} \mid \mathrm{E}[Z]=0,\|Z\|_{2}^{2}=1\right\}
$$


This allows us to write (see Sect. 3.4.2 in [11]) the minimization problem of firm $i$ as that of finding a stationary point to the Lagrangian

$$
L(Z, \tau, \kappa)=\ln \left(\int_{\Omega} e^{-\gamma_{i}\left(W+Z a\left(v_{i}, f_{i}\right)\right)} d \mathbb{P}\right)+\tau \int_{\Omega} Z d \mathbb{P}+\kappa \int_{\Omega} Z^{2} d \mathbb{P} .
$$

Here $\tau$ and $\kappa$ are the Lagrange multipliers associated to the moment constraints, and

$$
a\left(v_{i}, f_{i}\right)=\int_{\Theta_{i}} \sqrt{-v_{i}^{\prime}(\theta)} \mu(d \theta)+\int_{\Theta_{0}} \sqrt{-v_{i}^{\prime}(\theta)} f_{i}(\theta) \mu(d \theta) .
$$

We have the following implicit representation for the optimal claims given $v_{i}$ and $f$, which has a unique solution for each realization $z_{i}^{v, f}$ of $Z_{i}^{v, f}$ and $w_{i}$ of $W_{i}$ :

$$
Z_{i}^{v, f}=-\frac{e^{-\gamma_{i}\left(W_{i}+Z_{i}^{v, f} a\left(v_{i}, f_{i}\right)\right)}-\mathrm{E}\left[e^{-\gamma_{i}\left(W_{i}+Z_{i}^{v, f} a\left(v_{i}, f_{i}\right)\right)}\right]}{\sqrt{\operatorname{Var}\left(e^{-\gamma_{i}\left(W_{i}+Z_{i}^{v, f} a\left(v_{i}, f_{i}\right)\right)}\right)}} .
$$

Remark 5.1 The indirect utility functions $v_{i}$ and the TBR $f_{i}$ only affect the optimal claims $Z_{i}^{v, f}$ via the "aggregator" $a\left(v_{i}, f_{i}\right)$.

Remark 5.1 has interesting repercussions for the socially efficient TBRs. Indeed, assume the firms have initial endowments $W_{i}$ and they are characterized by risk aversion coefficients $\gamma_{i}$. Theorem 4.10 guarantees the existence of a SEA $\left(v_{1}^{*}, v_{2}^{*}, f^{*}, Z_{1}^{*}, Z_{2}^{*}\right)$, which yields the following aggregate risk in the economy:

$$
\varrho_{1}\left(W_{1}-a\left(v_{1}^{*}, f_{1}^{*}\right) Z_{1}^{*}\right)+\varrho_{2}\left(W_{2}-a\left(v_{2}^{*}, f_{2}^{*}\right) Z_{2}^{*}\right)+I\left[v_{1}^{*}, v_{2}^{*}\right]
$$

where $I\left[v_{1}^{*}, v_{2}^{*}\right]$ represents the firms' aggregate income (which is independent of $f^{*}$ ). We know from Remark 5.1 that any modification on $f^{*}$ that leaves $a\left(v_{i}^{*}, f_{i}^{*}\right)$ unchanged bears no weight on the value of expression (7). We can redefine

$$
f^{*}(\theta)= \begin{cases}0, & v_{1}(\theta)<v_{2}(\theta) \\ 1, & v_{1}(\theta)>v_{2}(\theta) \\ f^{*}(\theta), & \text { otherwise }\end{cases}
$$

and define

$$
v^{*}(\theta) \triangleq \max \left\{v_{1}^{*}(\theta), v_{2}^{*}(\theta)\right\}
$$

This allows us to write

$$
a\left(v_{i}^{*}, f_{i}^{*}\right)=\int_{\Theta} \sqrt{-\left(v^{*}\right)^{\prime}(\theta)} f_{i}^{*}(\theta) \mu(d \theta) .
$$

Once we have defined the TBR over $\Theta$, we may go one step further and write

$$
K \triangleq \frac{\int_{\theta} \sqrt{-\left(v^{*}\right)^{\prime}(\theta)} f^{*}(\theta) \mu(d \theta)}{\int_{\theta} \sqrt{-\left(v^{*}\right)^{\prime}(\theta)} \mu(d \theta)},
$$

then

$a\left(v_{1}^{*}, f_{1}^{*}\right)=K \int_{\Theta} \sqrt{-\left(v^{*}\right)^{\prime}(\theta)} \mu(d \theta)$ and $a\left(v_{2}^{*}, f_{2}^{*}\right)=(1-K) \int_{\Theta} \sqrt{-\left(v^{*}\right)^{\prime}(\theta)} \mu(d \theta)$.

An interesting economical conclusion from the computations above is that there exists a SEA that consists of both firms servicing the whole market, and splitting the customers in 
a $K$ to $1-K$ proportion. This follows from the fact that only $v^{*}$ (the upper envelope of the original $v_{i}^{*}$ ) appears in each firm's program. In markets such as regulated health-insurance, where firms are legally prevented from abstaining from contracting with any agent, the regulator may oversee that a socially optimal proportion of the market is serviced by each firm.

\subsection{Simulations (Entropic-risk minimizing firms)}

In this section we provide the numerical analysis of a particular example of the entropic-risk minimizing firms analyzed above. To this end we set

- Dimension of the space defining $v: 6$, dimension of $\Omega: 14$,

- $W_{1}=0.5 *(-1,-3,-9,-3,-1,-0.2,-0.1,-0.1,-0.2,1,-3,-9,-3,-1)^{T}$,

- $W_{2}=0.5 *(-0.03,-0.1,-0.18,-0.2,-1,-3,-9,-10,-3,-1,-0.2,-0.18,-0.1$, $-0.03)^{T}$

- risk aversion coefficient $\gamma=2$.

\subsubsection{Risk assessments}

Let us benchmark the aggregate risk in the competitive economy against the risk in a monopolistic setting. We first fix $f \equiv 1$. This corresponds to a model in which firm 1 acts as a monopolist and firm 2 has no access to the market. The a-priori aggregate risk in the economy is 7.36. The risk assessment of firm 1 is reduced from 3.53 to 2.16 after it has traded with the agents. The risk of firm 2 remains unchanged. Below we plot the numerical result for $Z$, as well as the theoretical one from Eq. (6) in Fig. 1a and the corresponding minimizing $v$ (the agents' indirect utility) in Fig. 1b. Likewise, if we fix $f \equiv 0$, which again yields a principal-agent setting for firm 2, we obtain that this firm's initial risk is 3.84 , which is reduced to 2.30 after trading. Finally, once we let $f$ vary, the aggregate risk decreases from 7.36 to 5.39, and the corresponding final risk assessments for the firms are 2.17 and 2.67 respectively. Each firm is worse off in the presence of competition, but aggregate risk in the economy is lower. Risk decreases from 7.36 to 5.99 when only firm 1 is active, and from 7.36 to 5.72 if it is firm 2 who trades with the agents.

\subsubsection{Risk profiles}

With respect to the ex-ante and ex-post risk profiles, we observe that trading has a smoothing effect, flattening spikes that correspond to the bad states of the World. However the basic shape of the risk profile remains, which is in contrast to what we find in Fig. 4 of our AV@ $R$ example. This is presented in Fig. 2, where the 14 elementary events have been connected by lines for illustration purposes. Figure 3 shows the agents' indirect utilities associated to each firm's offer, and compares it to the indirect utility for the agents who face a monopolist (in this case firm 1). The market is shared at a 0.42 to 0.58 ratio between the firms. We observe that the upper envelope $v(\theta)=\max \left\{v_{1}(\theta), v_{2}(\theta)\right\}$ dominates the monopolistic situation for all agents. This example is in line with the intuition that competition among sellers benefits the buyers. A point could be made that the regulator should make sure that enough incentives exist for all firms to engage in risk-minimizing trading, as it is socially desirable.

Remark 5.2 Arguably, the implementation of an efficient TBR in full generality could prove to be a daunting task. However, the structure of $Z_{i}^{v, f}$ presented in Eq. (6) suggests that in 


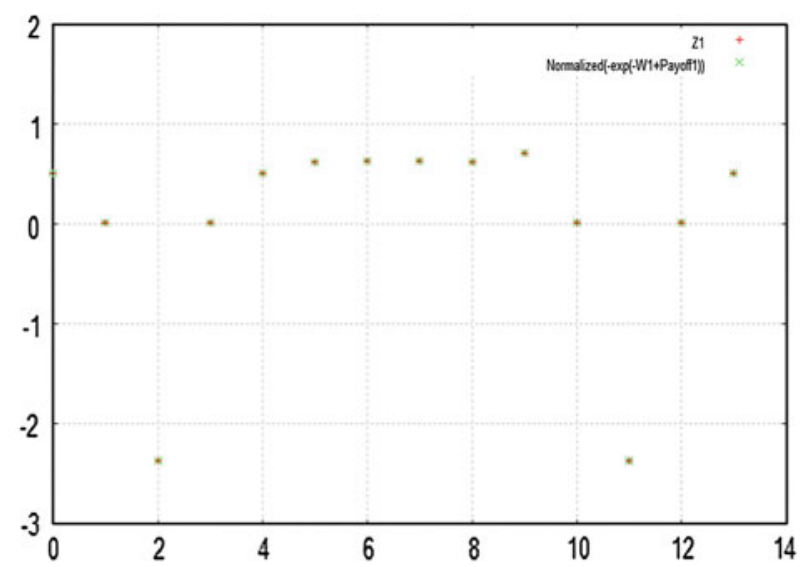

(a) Numerical v.s. theoretical values for $Z$

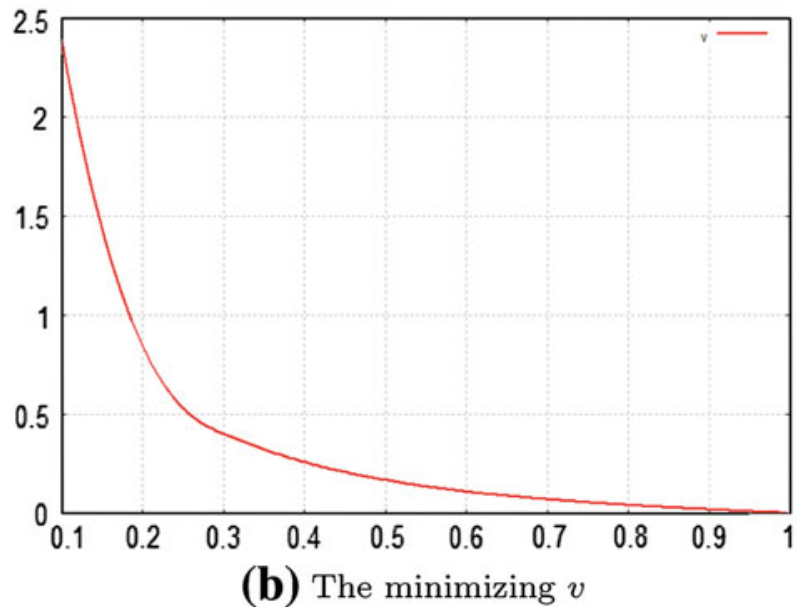

Fig. 1 Comparison results for the $f \equiv 1$ case

general these variables depend on $v$ and $f$ only via the integral expression $a(v, f)$. If such were the case, one could achieve an optimum by choosing an efficient "fix-mix" TBR, where each firm caters to a constant proportion of the whole market. The latter clearly makes the implementation considerably simpler.

\subsection{Simulations (AV@R-minimizing firms)}

In this section we study an example where the firms are average value-at-risk minimizers. Recall that for $\lambda \in(0,1]$, and $X \in \mathbb{L}^{\infty}(\Omega, \mathbb{F}, P)$, one defines

$$
A V @ R_{\lambda}(X) \triangleq \frac{1}{\lambda} \int_{0}^{\lambda} V @ R_{\gamma}(X) d \gamma,
$$

where

$$
V @ R_{\gamma}(X) \triangleq \inf \{m \mid P\{X+m<0\} \leq \gamma\}
$$




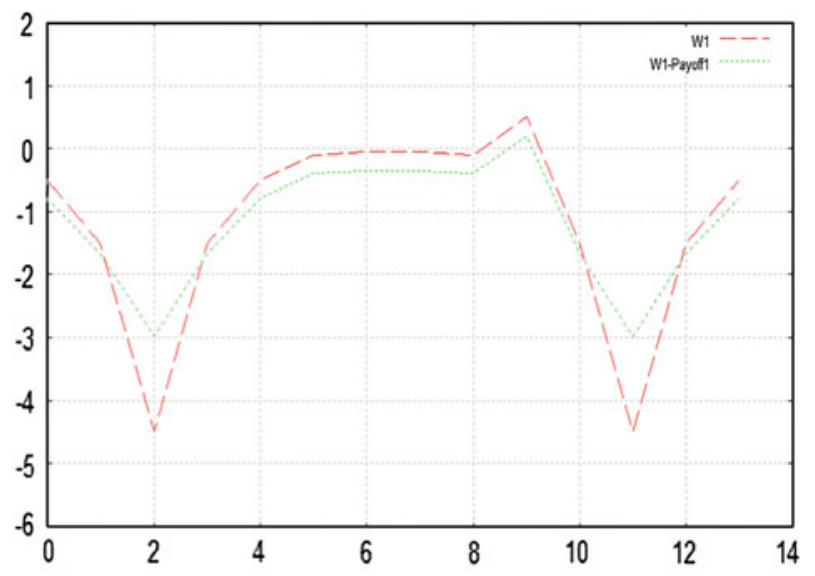

(a) Comparison of $W_{1}$ and $W_{1}-a\left(v_{1}^{*}, f_{1}^{*}\right) Z_{1}^{*}$

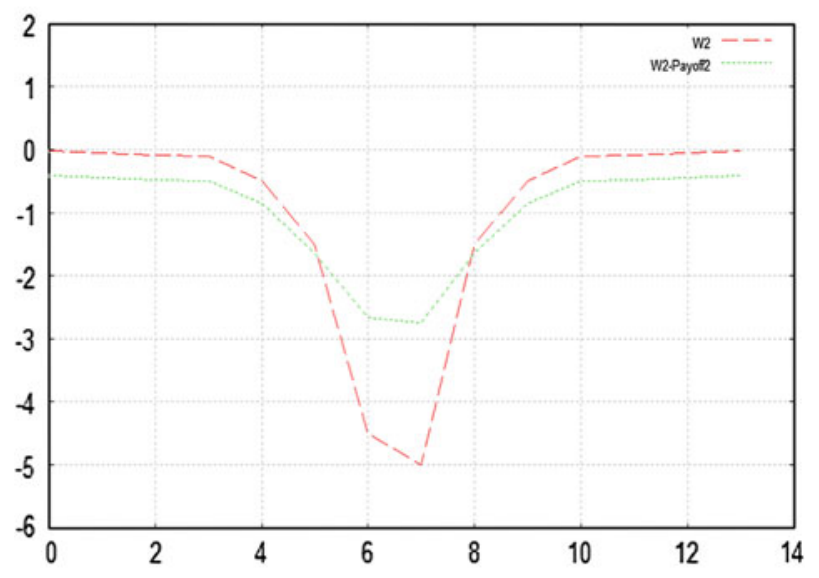

(b) Comparison of $W_{2}$ and $W_{2}-a\left(v_{2}^{*}, f_{2}^{*}\right) Z_{2}^{*}$

Fig. 2 Positions before and after trading

We use the following initial parameters:

- Dimension of the space defining $v: 6$, dimension of $(\Omega): 14$,

- $W_{1}=0.02 *(-1,-2,-4,-10,-4,-2,-1,-0.8,-0.5,-0.3,0,0,0,0)^{T}$,

- $W_{2}=0.05 *(-0.03,-0.1,-0.18,-0.2,-1,-3,-9,-10,-3,-1,-0.2,-0.18$, $-0.1,-0.03)^{T}$,

- levels for the AV@R: $\lambda_{1}: 0.05$ and $\lambda_{2}: 0.1$

The initial aggregate initial risk assessment is 0.68 , which decreases to 0.21 after trading. In Fig. 4 we compare the firms' positions before and after trading. We observe that in contrast with the entropic-risk-measure case (see Fig. 2), the ex-post shapes of the risk profiles have been significantly altered. This is due to the fact that the risk measure in hand places a heavier weight on the bad states of nature, even at the cost of the originally good ones. Figure 5a 


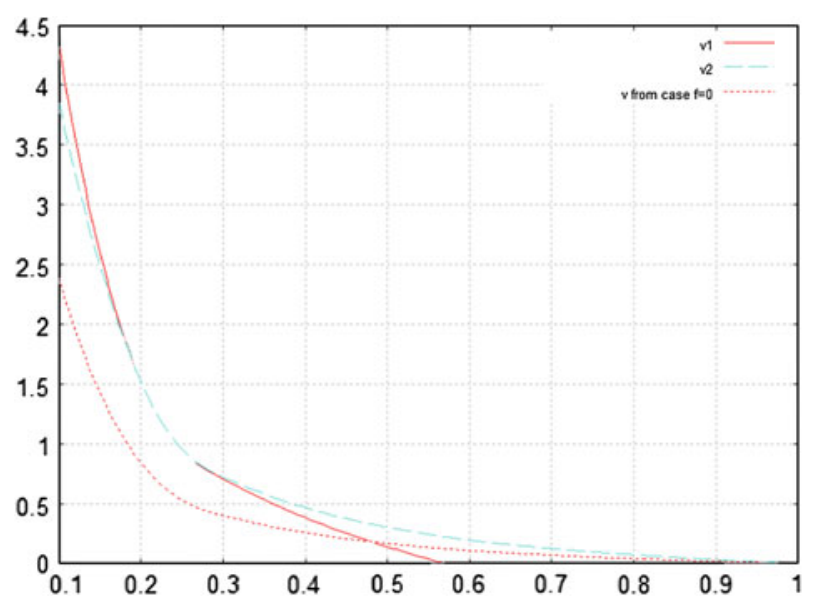

Fig. 3 Indirect utilities under monopoly and oligopoly

shows the indirect utility functions corresponding to the catalogue that each firm offers, and in Fig. 5b we have plotted an efficient TBR.

Remark 5.3 It should be noted that in neither of the examples presented above were the individual rationality constraints for the firms binding. This is essential to implement the "fix-mix" TBR without the need of a cash transfer. Although $f$ plays no role in the aggregate income $I\left[v_{1}, v_{2}\right]$, it does enter the IR constraints of each firm. If one wanted to implement a "fix-mix" TBR without a cash transfer scheme, they would in general run into a two-equations-one-unknown issue as soon as an IR constraint bound.

\section{Conclusions}

We have extended the principal-agent models of profit maximization found in [6] and risk minimization found in [11] to a multi-firm setting. Both of these works deal with overthe-counter trading of derivatives under adverse selection. On the profit maximization side, we have used results of Page Jr. and Monteiro([17-19] and [20]). These authors, together with Bagh and Jofré ([3]), provide testable conditions that allow for Reny's results to be used in multi-firm-agent games. We have shown the existence of (mixed-strategies) Nash equilibria when efficient tie breaking rules are used (and most likely enforced by a regulator). Our contribution on risk minimization follows on the footsteps of Barrieu and El Karoui's ([4]), and Jouini, Schachermayer and Touzi's ([12]) work on optimal risk sharing for convex risk measures. In [12] it is shown that optimal allocations are Pareto optimal but not necessarily individually rational. A cash transfer, also called the rent of risk exchange, could be necessary to address this issue. Implementing a cash transfer and/or imposing socially efficient tie breaking rules are the raisons d'être for a regulator in our model. We did not prove (as in the profit-maximization case) that the game is uniformly payoff secure and (weakly) reciprocal upper semicontinuous. Since the game is not quasi-convexity, we would have to consider its mixed extension. This brings us to the following conceptual issue: what is expected risk? Convex risk measures can be (robustly) represented as a worst-case analysis over a family of probability measures that are absolutely continuous with respect to the reference one. 


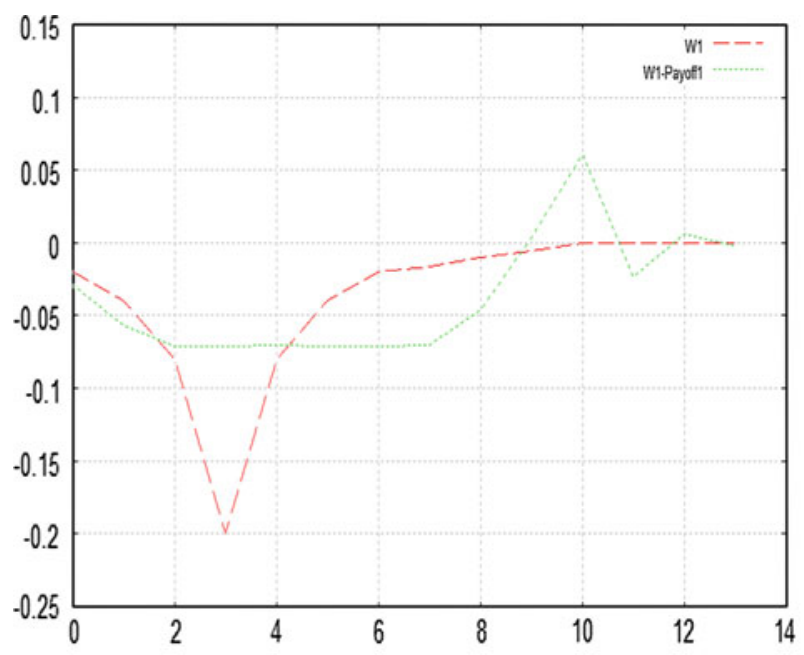

(a) Comparison of $W_{1}$ and $W_{1}-a\left(v_{1}^{*}, f_{1}^{*}\right) Z_{1}^{*}$

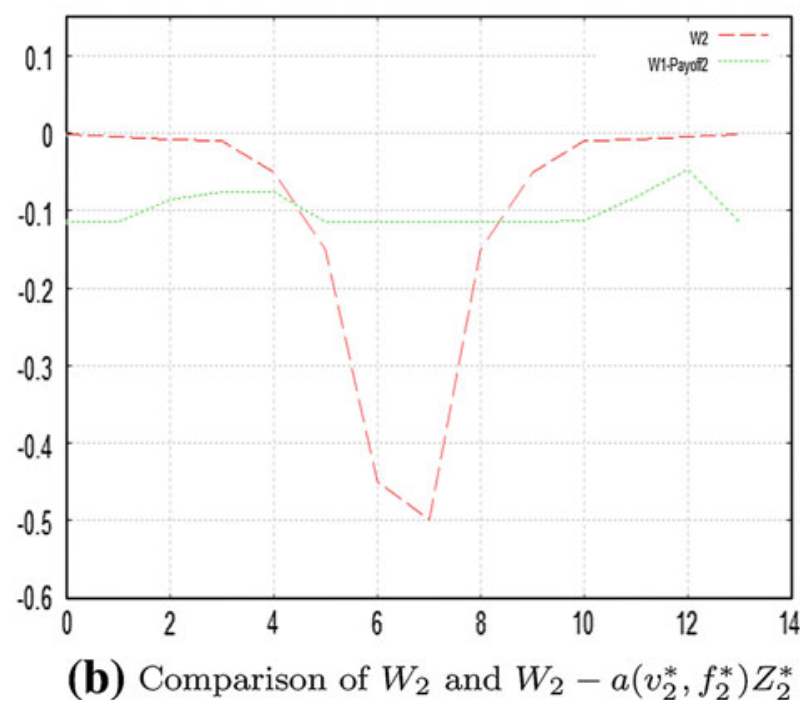

Fig. 4 Positions before and after trading

However, not all of these measures (which can be interpreted as possible distributions of the future states of the World) are given the same weight. Indeed, they are penalized according to a function that maps the space of probability measures into the extended Reals. By considering a mixed extension of our risk-minimization game, we implicitly assume that "Nature" and the firms behave in qualitatively different ways. In our view this would be inconsistent. Instead, we introduced the notion of socially efficient allocations and proved the existence of such. We believe that an important contribution of this paper is to show that, within our stylized setting, non-regulated, over-the-counter markets cannot be guaranteed to be efficient (in the sense of the welfare theorems). The extension of our general setting to one where agents 


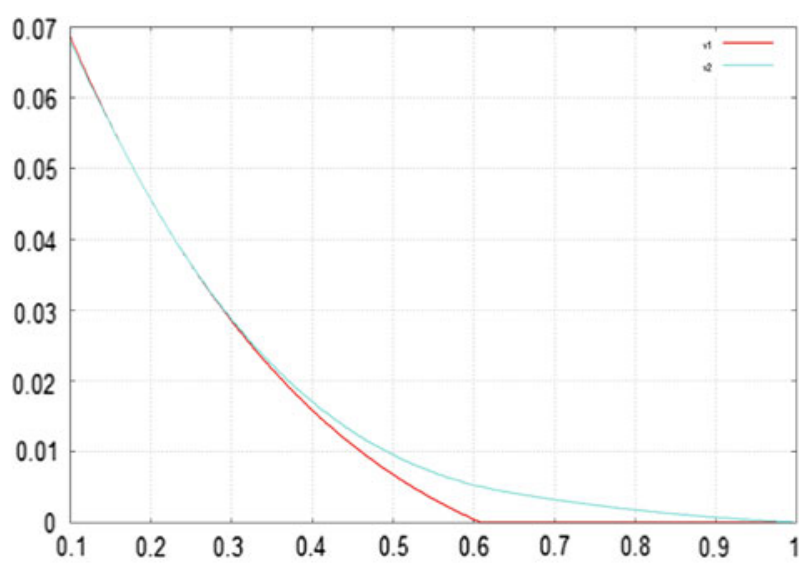

(a) The indirect utility functions

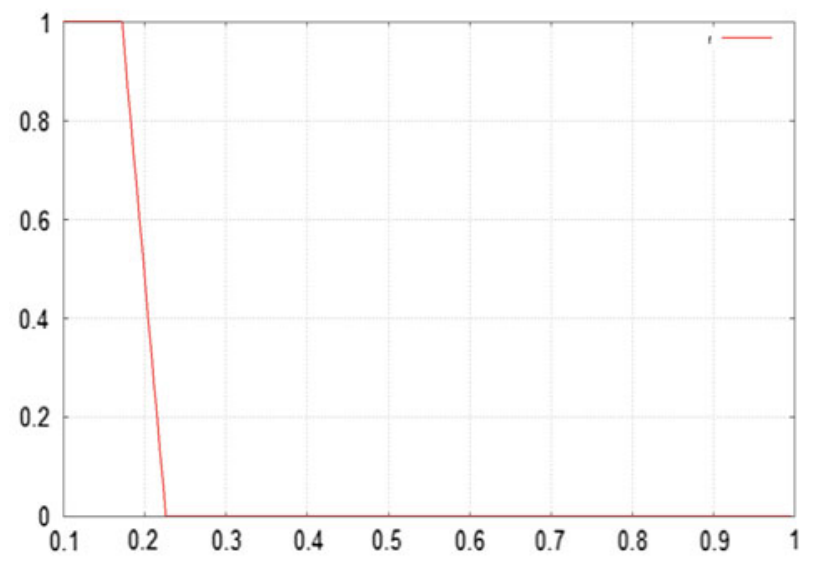

(b) A possible socially efficient TBR

Fig. 5 Indirect utilities and a possible efficient TBR

have heterogenous initial endowments (multi-dimensional agent types) and (we believe more interestingly) to a dynamic framework are left for future research.

Acknowledgements We thank Guillaume Carlier, Ivar Ekeland, and seminar participants at various institutions for valuable comments and suggestions. We are grateful to Alexander Fromm for his help in developing Sect. 5 of this work. This paper was finalized while the authors were visiting the Institute for Mathematical Sciences at the National University of Singapore. Financial support from the Deutsche Forschungsgemeinschaft through the SFB 649 "Economic Risk" and from the Alexander von Humbold Foundation via a research fellowship is gratefully acknowledged. Finally, would like to acknowledge two anonymous referees, whose thorough reading and suggestions led to an improved version of our original manuscript.

\section{Appendix A: Proofs}

Proof of Proposition 2.3: When firm $i$ designs a product line, it takes into account that

$$
-M^{2} \leq \mathrm{E}[X]-\theta \operatorname{Var}[X] \leq M .
$$


Therefor all $p \in P$ must be below $M$ to satisfy the individual rationality constraint. In counterpart $-M^{2} \leq p$, otherwise all agents would be guaranteed an indirect utility above their reservation utility. Since $\|X\|_{2} \leq M$ for all $X \in \mathcal{X}_{i}$, then $\left|v_{i}^{\prime}\right| \leq M$ for all $v_{i} \in \mathcal{C}_{i}$, thus $0 \leq v_{i} \leq 3 \cdot \max \left\{M, M^{2}\right\}$. The convexity of the elements of the (closed) set $\mathcal{C}_{i}$ implies they are locally Lipschitz (see for example [21]); moreover, the $\mathbb{L}^{2}$-boundedness of $\mathcal{X}_{i}$ together with Eq. (1) imply the Lipschitz coefficients are uniformly bounded. This in turn means that $\mathcal{C}_{i}$ is a bounded, closed and uniformly equicontinuous family, which by the Arzelà-Ascoli is then compact for the topology of uniform convergence.

Proof of Proposition 3.1: Consider $(\bar{X}, \bar{p}) \in \operatorname{cl}\left(\bigcup_{\theta} \mathcal{L}\left(\theta, C_{i}\right)\right)$, then there exists a sequence $\left\{\left(X_{n}, p_{n}\right)\right\} \subset \bigcup_{\theta} \mathcal{L}\left(\theta, C_{i}\right)$ such that $\left(X_{n}, p_{n}\right) \stackrel{\|\cdot\|_{2} \times|\cdot|}{\longrightarrow}(\bar{X}, \bar{p})$. By construction $\left(X_{n}, p_{n}\right) \in$ $\mathcal{L}\left(\theta, C_{i}\right)$ for some $\theta \in \Theta$, call it $\theta_{n}$. Passing to a subsequence if necessary we may assume there is $\bar{\theta} \in \Theta$ such that $\theta_{n} \rightarrow \bar{\theta}$ and $\left(X_{n}, p_{n}\right) \rightarrow(\bar{X}, \bar{p})$ pointwise. If $(\bar{X}, \bar{p}) \in \bigcup_{\theta} \mathcal{L}\left(\theta, C_{i}\right)$ we are done, so let us assume the contrary, thus

$$
\mathrm{E}[\bar{X}]-\bar{\theta} \operatorname{Var}[\bar{X}]-\bar{p}<\mathrm{E}[X]-\bar{\theta} \operatorname{Var}[X]-p \quad \forall(X, p) \in \mathcal{L}\left(\bar{\theta}, C_{i}\right) .
$$

By definition $\mathrm{E}\left[X_{n}\right]-\theta_{n} \operatorname{Var}\left[X_{n}\right]-p_{n} \geq \mathrm{E}[X]-\theta_{n} \operatorname{Var}[X]-p \quad \forall(X, p) \in C_{i}$. However, the (a.s.) convergence of the sequence $\left\{\left(X_{n}, p_{n}\right)\right\}$ implies $\mathrm{E}[\bar{X}]-\bar{\theta} \operatorname{Var}[\bar{X}]-\bar{p} \geq$ $\mathrm{E}[X]-\bar{\theta} \operatorname{Var}[X]-p \quad \forall(X, p) \in C_{i}$. This would imply $(X, p) \in \mathcal{L}\left(\bar{\theta}, C_{i}\right)$, contradicting Eq. (8).

Proof of Lemma 3.5: Consider the mapping $\left(C_{1}, C_{2}\right) \mapsto \sum_{i} \Pi_{i}\left(f^{*}, C_{1}, C_{2}\right)$. If $f^{*}$ is efficient then

$$
\begin{aligned}
\sum_{i} \Pi_{i}\left(f^{*}, C_{1}, C_{2}\right) & =\sup _{f \in F} \sum_{i} \Pi_{i}\left(f, C_{1}, C_{2}\right) \\
& =\sup _{f \in F} \sum_{i} \int_{\Theta} \pi_{i}\left(\theta, C_{1}, C_{2}\right) f_{i}(\theta) \mu(d \theta) \\
& =\int_{\Theta} \max _{i} \pi_{i}\left(\theta, C_{1}, C_{2}\right) \sum_{i} f_{i}(\theta) \mu(d \theta) \text { for any } f \in F \\
& =\int_{\Theta} \max _{i} \pi_{i}\left(\theta, C_{1}, C_{2}\right) \mu(d \theta) .
\end{aligned}
$$

It follows from Proposition 3.2, that $\left(C_{1}, C_{2}\right) \mapsto \max _{i} \pi_{i}\left(\theta, C_{1}, C_{2}\right)$ is upper semicontinuous, hence so is $\sum_{i} \Pi_{i}\left(f^{*}, C_{1}, C_{2}\right)$.

Proof of Proposition 3.9: Let $C_{i} \in \mathcal{P}_{i}$ and $\epsilon>0$ and define

$$
C_{i}^{\epsilon} \triangleq\left\{(X, p-\epsilon) \mid(X, p) \in C_{i} \text { and } p-\epsilon \geq K_{i}(X)\right\} \cup\{(0,0)\} .
$$

Lemma 2.1 implies there is $\delta>0$ such that if $d\left((X, p),\left(X^{\prime}, p^{\prime}\right)\right)<\delta$ (where $d(\cdot, \cdot)$ is the distance generated by $\left.\|\cdot\|_{2} \times|\cdot|\right)$ then for all $\theta \in \Theta$

$$
\left|U(\theta, X)-p-U\left(\theta, X^{\prime}\right)+p^{\prime}\right|<\epsilon .
$$

Assume first that $\pi_{i}\left(\theta, C_{i}, C_{-i}\right)>\epsilon$ and let $(X, p) \in C_{i}$ be such that $U(\theta, X)-p=$ $\pi_{i}\left(\theta, C_{i}, C_{-i}\right)$. Then $(X, p-\epsilon) \in C_{i}^{\epsilon}$. Now consider $C_{-i}^{\prime}$ such that $h_{p}\left(C_{-i}, C_{-i}^{\prime}\right)<\delta$, and let $\left(X^{\prime}, p^{\prime}\right) \in C_{-i}^{\prime}$. By definition there exist $(Y, q) \in C_{-i}$ such that $d\left((Y, q),\left(X^{\prime}, p^{\prime}\right)\right)<\delta$, and by Eq. (9)

$$
U(\theta, X)-(p-\epsilon) \geq U(\theta, Y)-(q-\epsilon)>U\left(\theta, X^{\prime}\right)-p^{\prime} .
$$


The latter implies that $\left(X^{\prime}, p^{\prime}\right) \notin \mathcal{L}\left(\theta, C_{i}^{\epsilon}, C_{-i}^{\prime}\right)$ and in turn that an efficient TBR $f$ for $\left(C_{i}^{\epsilon}, C_{-i}^{\prime}\right)$ satisfies $f_{i}(\theta)=1$. Thus $\pi_{i}\left(\theta, C_{i}^{\epsilon}, C_{-i}^{\prime}\right) f_{i}(t) \geq \pi_{i}\left(\theta, C_{i}^{\epsilon}, C_{-i}^{\prime}\right)$. The inequality above is trivially fulfilled if $\pi_{i}\left(\theta, C_{i}, C_{-i}\right) \leq \epsilon$. We have that for any deviation $C_{-i}^{\prime}$ such that $h_{p}\left(C_{-i}, C_{-i}^{\prime}\right)<\delta$ and for any efficient TBR $f$

$$
\Pi_{i}\left(C_{i}^{\epsilon}, C_{-i}^{\prime}\right) \geq \int_{\Theta} \pi_{i}\left(\theta, C_{i}^{\epsilon}, C_{-i}^{\prime}\right) f_{i}(\theta) \mu(d \theta)-\epsilon=\Pi_{i}\left(C_{i}, C_{-i}\right)-\epsilon .
$$

Proof of Proposition 4.2: The set $\Theta_{s}\left(v_{1}, v_{2}\right)$ is the union of the closures of the sets

$$
\Theta_{s}^{1} \triangleq\left\{\theta \in \Theta \mid v_{1}(x)=v_{2}(x), v_{1}^{\prime}(x) \neq v_{2}^{\prime}(x)\right\}
$$

and $\Theta_{s}^{\epsilon} \triangleq\left\{\theta \in \Theta\left|v_{1}(x)=v_{2}(x), v_{1}^{\prime}(x)=v_{2}^{\prime}(x),\right| v_{1}(\tilde{x})-v_{2}(\tilde{x}) \mid>0 \forall \tilde{x} \in B(\epsilon, x) \backslash\right.$ $\{x\}, \epsilon>0\}$. The set $\Theta_{s}^{1}$ is denumerable and nowhere dense. This follows directly from the convexity of $v_{1}$ and $v_{2}$ and the fact that the corresponding supporting planes to graph $\left\{v_{1}\right\}$ and graph $\left\{v_{2}\right\}$ at $\left(\theta, v_{1}(\theta)\right)$ are secant. By definition $\Theta_{2}$ is denumerable and nowhere dense, since any two of its elements can be separated. Therefore $\operatorname{cl}\left\{\Theta_{s}^{1}\right\}$ and $\operatorname{cl}\left\{\Theta_{s}^{\epsilon}\right\}$ are themselves denumerable and nowhere dense.

Proof of Lemma 4.7: Adding and subtracting $\left\langle\phi, \psi_{n}\right\rangle$ from $\left|\left\langle\phi_{n}, \psi_{n}\right\rangle-\langle\phi, \psi\rangle\right|$ we obtain

$$
\left|\left\langle\phi_{n}, \psi_{n}\right\rangle-\langle\phi, \psi\rangle\right| \leq\left|\left\langle\phi_{n}-\phi, \psi_{n}\right\rangle\right|+\left|\left\langle\phi, \psi_{n}-\psi\right\rangle\right| .
$$

Since $\left\{\psi_{n}\right\}$ is a weakly convergent sequence, it is bounded. Let $\bar{K}$ be such bound, then using the Cauchy-Schwarz inequality we have $\left|\left\langle\phi_{n}, \psi_{n}\right\rangle-\langle\phi, \psi\rangle\right| \leq \bar{K}\left\|\phi_{n}-\phi\right\|_{2}+\left|\left\langle\phi, \psi_{n}-\psi\right\rangle\right|$. As $n \rightarrow \infty$, the first summand on the righthand side of the inequality converges to zero due to the strong convergence of the $\phi_{n}$ 's to $\phi$; the second summand converges to zero due to the weak convergence of the $\psi_{n}$ 's to $\psi$, which concludes the proof.

Proof of Proposition 4.8: The second inequality follows from the definition of $\left(\bar{X}_{1}, \bar{X}_{2}, \bar{f}\right)$. To show that the first one holds, we first use Proposition 4.6 and write

$$
\begin{aligned}
& \int_{\Theta_{i}^{n}} X_{i}^{v^{n}, f^{v^{n}}} \mu(d \theta)-\int_{\Theta_{0}^{n}} f_{i}^{n} X_{i}^{v^{n}, f^{v^{n}}} \mu(d \theta) \\
& =Z_{i}^{v^{n}, f^{v^{n}}} \int_{\Theta}\left(11_{\Theta_{i}^{n}}(\theta)-f_{i}^{v^{n}} 11_{\Theta_{0}^{n}} V_{i}^{n}(\theta)\right) \mu(d \theta) \triangleq a_{i}^{v^{n}} \cdot Z_{i}^{v^{n}, f^{v^{n}}} .
\end{aligned}
$$

The assumption on the convergence of the indicator functions $11_{\Theta_{j}^{n}}$ implies that $V_{i}^{n} 1_{\Theta_{0}^{n}} \stackrel{\text { a.s. }}{\longrightarrow}$ $\bar{V}_{i} 11_{\bar{\Theta}_{0}}$. Since $\left|V_{i}^{n} 11_{\Theta_{0}^{n}}\right|,\left|\bar{V}_{i} 11_{\bar{\Theta}_{0}}\right| \leq M$ and $\mu(\Omega)<\infty$, by Lebesgue Dominated Convergence we get $V_{i}^{n} 11_{\Theta_{0}^{n}} \stackrel{\|\cdot\|_{2}}{\longrightarrow} \bar{V}_{i} 11_{\Theta_{0}}$. Hence, by Lemma 4.7 we have that $a_{i}^{v^{n}} \rightarrow \bar{a}_{i}$, where

$$
\bar{a}_{i} \triangleq \int_{\Theta}\left(11_{\Theta_{1}}(\theta)-\bar{f} 11_{\Theta_{0}} \bar{V}_{i}(\theta)\right) \mu(d \theta) .
$$

Therefore $a_{i}^{v^{n}} \cdot Z_{i}^{v^{n}} \stackrel{w}{\rightarrow} \bar{a}_{i} \cdot \bar{Z}_{i}$, and the Fatou property of the risk measure yields

$$
\sum_{i=1}^{2} R_{i}\left(\bar{v}_{1}, \bar{v}_{2}, X_{i}, f\right) \leq \liminf _{n \rightarrow \infty} \sum_{i=1}^{2} \tilde{R}_{i}\left(v_{1}^{n}, v_{2}^{n}, f^{v^{n}}\right) .
$$


Proof of Lemma 4.9: Let $\theta \in \Theta$ be given. There are two possible cases. Either there exists $N \in \mathbb{N}$ such that if $n>N$, then $\theta \in \tilde{\Theta}_{i}^{n}$ or there exists a subsequence $\left\{v_{1}^{n_{k}}, v_{2}^{n_{k}}\right\}$ such that $\theta \notin \tilde{\Theta}_{i}^{n_{k}}$ for all $n_{k}$. In the former case the (uniform) convergence of the $v_{i}^{n}$ 's implies $\bar{v}_{i}(\theta)>\bar{v}_{-i}(\theta)$. In the latter case, either $\theta$ eventually belongs to $\tilde{\Theta}_{-i}^{n}$, in which case convergence of the indicator functions follows or $\theta \in \tilde{\Theta}_{1}^{n} \cap \tilde{\Theta}_{2}^{n}$ infinitely often. In this case $\theta \in \tilde{\Theta}_{0}^{n}$ and we again have convergence of the indicators.

\section{Appendix B: Existence of minimizers to $\Psi_{1}$}

In this appendix we give an overview of the proof of existence of minimizers to problem $\Psi_{1}$ in Sect. 4.2.1, which is analogous to the proof of Theorem 2.3 in [11]. The steps to be taken are the following:

1. We fix $\left(v_{1}, v_{2}\right) \in \mathcal{C}_{1} \times \mathcal{C}_{2}$ and relax the variance constraint to $-\operatorname{Var}\left[X_{i}(\theta)\right] \leq v_{i}^{\prime}(\theta)$. We observe that $\left\|X_{i}\right\|_{2}^{2}=v_{i}(a)-v_{i}(0) \triangleq k_{i}^{v}$. Let $K_{i}$ be the uniform bound on the elements of $\mathcal{C}_{i}$ (see Prop. 2.3), then $\left\|X_{i}\right\|_{2}^{2} \leq k_{i}^{v} \leq K_{i}$.

2. The convexity of $\varrho_{i}$ implies that the set $\mathcal{X}_{i}^{v, f} \triangleq\left\{X_{i} \in \mathcal{X}_{i} \mid A_{i}\left(v_{1}, v_{2}, X_{i}, f\right) \leq\right.$ $\left.\varrho_{i}\left(W_{i}\right)\right\}$ is convex. The fact that $\rho_{i}$ has the Fatou property implies that $\mathcal{X}_{i}^{v, f}$ is closed.

3. Since the mapping

$$
X_{i} \mapsto \int_{\Theta_{i}} X_{i}(\theta) d \theta-\int_{\Theta_{0}} X_{i}(\theta) f_{i}(\theta) d \theta
$$

is a linear, then $X_{i} \mapsto A_{i}\left(v_{1}, v_{2}, X_{i}, f\right)$ is a convex mapping. The set $\mathcal{X}_{i}^{k_{i}^{v}} \triangleq\{X \in$ $\left.\mathcal{X}_{i} \mid\left\|X_{i}\right\|_{2}^{2} \leq k_{i}^{v}\right\}$ is closed, convex and bounded. Therefore the relaxed optimization problem has a solution $\tilde{X}_{i}^{v, f}$, as long as $\mathcal{X}_{i}^{K} \cap \mathcal{X}_{i}^{v, f} \neq \varnothing$.

4. The variance constraint can be made binding by structuring the products $\tilde{X}_{i}^{v}$ into $X_{i}^{v, f}=$ $\tilde{X}_{i}^{v, f}+\alpha Y$. Here $Y$ is independent of $\theta$ and $\alpha: \Theta \rightarrow \mathbb{R}$ integrates to zero.

\section{Description of the algorithm used in Sects. 5.2 and 5.3}

In this appendix we provide a brief description of our numerical algorithm, whose aim is to estimate solutions to the problem

$$
\inf _{(Z, f, v)} \sum_{i=1}^{2}\left[\varrho_{i}\left(W_{i}-Z_{i} \int_{a}^{1} \sqrt{-v^{\prime}(\theta)} f_{i}(\theta) d \theta\right)+\int_{a}^{1}\left(v(\theta)-\theta v^{\prime}(\theta)\right) f_{i}(\theta) d \theta\right]
$$

subject to:

- $f_{i} \geq 0, f_{1}+f_{2}=1 ; v$ convex, $v \geq 0, v^{\prime} \leq 0, v(1)=0 ; \mathrm{E}\left[Z_{i}\right]=0, \operatorname{Var}\left[Z_{i}\right]=1$;

- $\varrho_{i}\left(W_{i}-Z_{i} \int_{a}^{1} \sqrt{-v^{\prime}(\theta)} f_{i}(\theta) d \theta\right)+\int_{a}^{1}\left(v(\theta)-\theta v^{\prime}(\theta)\right) f_{i}(\theta) d \theta \leq \varrho_{i}\left(W_{i}\right)$. It should be noted that in the examples presented in Sect. 5, this constraint was not binding.

In order to do so, we set a discretization level $n$, and we work with the following structures:

- $f_{1}(\lambda) \triangleq \sum_{k=0}^{n-1} \lambda_{k} \mu_{\left[a+k \frac{1-a}{n}, a+(k+1) \frac{1-a}{n}\right]}, f_{2}=1-f_{1}, 0 \leq \lambda_{i} \leq 1$. 
- $v^{\prime}(\cdot) \triangleq\left(\int_{.}^{1}-\frac{d}{d \theta} \sqrt{-v^{\prime}(\theta)} d \theta+\sqrt{-v^{\prime}(1)}\right)^{2}$, we shall denote $\tilde{v} \triangleq-\frac{d}{d \theta} \sqrt{-v^{\prime}(\theta)}$, and $\gamma \triangleq \sqrt{-v^{\prime}(1)} \geq 0$. There is a one-to-one correspondence between $\tilde{v} \geq 0, \gamma \geq 0$ and functions $v$ that satisfy the requirements of being convex, non-increasing and non-negative.

- We go one step further and define $\tilde{v}(\alpha) \triangleq \sum_{k=0}^{n-1} \alpha_{k} \|_{\left[a+k \frac{1-a}{n}, a+(k+1) \frac{1-a}{n}\right]}, \alpha_{i} \geq 0$.

- $Z_{i} \triangleq\left(\beta_{0}^{i}, \ldots, \beta_{d-1}^{i}\right) \in \mathbb{R}^{d}, \frac{1}{d} \sum_{k=0}^{d-1} \beta_{k}^{i}=0, \frac{1}{d} \sum_{k=0}^{d-1}\left(\beta_{k}^{i}\right)^{2} \leq 1$. Following the results presented in Appendix B, we have relaxed $\operatorname{Var}\left[Z_{i}\right]=1$ to $\operatorname{Var}\left[Z_{i}\right] \leq 1$.

In this setting we have that

$$
\varrho_{i}\left(W_{i}-Z_{i} \int_{a}^{1} \sqrt{-v^{\prime}(\theta)} f_{i}(\theta) d \theta\right)=r_{i}(\alpha, \beta, \gamma, \lambda) .
$$

and the problem amounts to minimizing the aggregate risk $\sum_{i=1}^{2} r_{i}(\alpha, \beta, \gamma, \lambda)$ subject to $r_{i}(\alpha, \beta, \gamma, \lambda) \leq r_{i}(0,0,0,0)$ and subject to constraints on $\alpha, \beta, \gamma$ and $\lambda$ specified earlier. The latter are convex (in fact most of them are linear)in the corresponding variables. A crucial property of the functions $r_{i}: \mathbb{R}^{n+2 d+1+n} \rightarrow \mathbb{R}$ is that for any three fixed entries, they are convex on the remaining variable. We use an iterative algorithm that performs a one-step, first-order descent in each direction alternately, such that the aggregate risk decreases in each step. At each of the steps we encounter the problem of minimizing a convex function with respect to convex constraints. Our coding has been done in Java, and we have used the NetBeans IDE 6.8 environment. In the case of $A V @ R$, we have used the or 124.jar package (OR-Objects 1.2.4) to calculate the required gradients. The descent procedures are all based on a local linearization of the function to be minimized, as well as of the constraints. Since all of these objects are convex, they can be locally approximated by the corresponding subgradients. The linearized function is minimized, subject to the linearized constraints, on a cubic neighborhood of the current point. This reduces to a linear optimization problem. Again the linear optimization package included in or 124.jar is used to obtain a minimizer. If this point does not satisfy the constraints (we carry the linearization error), a correction procedure is performed to obtain a feasible point close to the prior one (See Fig. 6). Finally, it is verified whether at this feasible point the value of the objective function has decreased.

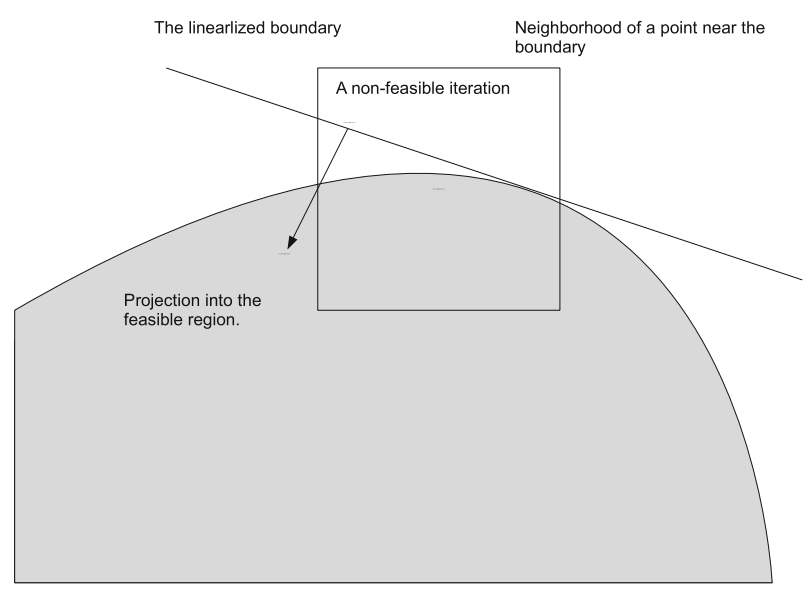

Fig. 6 Dealing with non-feasible points 
Otherwise, the procedure is repeated starting with a cube whose size length is half of the original one.

\section{References}

1. Aliprantis, C., Border, K.: Infinite Dimensional Analysis, a Hitchhiker's guide, 3rd edn. Springer, New York (2006)

2. Armstrong, M.: Multiproduct nonlinear pricing. Econometrica 64, 51-75 (1996)

3. Bagh, A., Jofré, A.: Reciprocal upper semicontinuity and better reply secure games, a comment. Econometrica 74, 1715-1721 (2006)

4. Barrieu, P., El Karoui, N.: Inf-convolution of risk measures and optimal risk transfer. Financ. Stoch. 9, 269-298 (2005)

5. Cheridito, P., Li, T.: Dual characterization of properties of risk measures in Orlicz hearts. Math. Financ. Econ. 2, 29-55 (2008)

6. Carlier, G., Ekeland, I., Touzi, N.: Optimal derivatives design for mean-variance agents under adverse selection. Math. Financ. Econ. 1, 57-80 (2007)

7. Dasgupta, P., Maskin, E.: The existence of equilibrium in discontinuous economic games, I: theory. Rev. Econ. Stud. 53, 1-26 (1986)

8. Ekeland, I., Moreno-Bromberg, S.: An algorithm for computing solutions of variational problems with global convexity constraints. Numer. Math. 115, 45-69 (2010)

9. Föllmer, H., Schied, A.: Stochastic Finance. An Introduction in Discrete Time. de Gruyter Studies in Mathematics, vol. 27 (2004)

10. Fudenberg, D., Tirole, J.: Game Theory. MIT Press, Cambridge (1991)

11. Horst, U., Moreno-Bromberg, S.: Risk minimization and optimal derivative design in a principal agent game. Math. Financ. Econ. 2, 1-27 (2008)

12. Jouini, E., Schachermeyer, W., Touzi, N.: Optimal risk sharing for law invariant monetary utility functions. Math. Financ. 18, 269-292 (2008)

13. Martimort, D., Stole, L.: The revelation and delegation principles in common agency games. Econometrica 70, 1659-1673 (2002)

14. Milgrom, P., Segal, I.: Envelope theorems for arbitrary choice sets. Econometrica 70, 583-601 (2002)

15. Mussa, M., Rosen, S.: Monopoly and product quality. J. Econ. Theory 18, 301-317 (1978)

16. Nessah, R., Tian, G.: The Existence of Equilibria in Discontinuous and Nonconvex Games. Working Paper, http://ideas.repec.org/p/ies/wpaper/e200814.html (2010)

17. Page, F. Jr..: Catalogue competition and stable nonlinear prices. J. Math. Econ. 44, 822-835 (2008)

18. Page, F. Jr., Monteiro, P.: Three principles of competitive nonlinear pricing. J. Math. Econ. 39, 63-109 (2003)

19. Page, F. Jr.., Monteiro, P.: Uniform payoff security and nash equilibrium in compact games. J. Econ. Theory 134, 566-575 (2007)

20. Page, F. Jr.., Monteiro, P.: Catalogue competition and nash equilibrium in nonlinear pricing games. Econ. Theory 34, 503-524 (2008)

21. Phelps R.: Convex Functions, Monotone Operators and Differentiability: Lecture Notes in Mathematics, vol. 1364. Springer, New York (1989)

22. Reny, P.: On the existence of pure and mixed strategy equilibria in discontinuous games. Econometrica 67, 1029-1056 (1999)

23. Rochet, J.-C., Choné, P.: Ironing, sweeping and multidimensional screening. Econometrica 66, 783-826 (1988)

24. Tian, G.: The Existence of Equilibria in Games with Arbitrary Strategy Spaces and Payoffs: A Full Characterization. Working paper, http://ideas.repec.org/p/cla/levarc/814577000000000160.html (2009) 